\title{
LINEAR PRESERVERS AND REPRESENTATIONS WITH A 1-DIMENSIONAL RING OF INVARIANTS
}

\author{
H. BERMUDEZ, S. GARIBALDI, AND V. LARSEN
}

\begin{abstract}
We determine the group of linear transformations preserving a polynomial function $f$ on a vector space $V$ for several interesting pairs $(V, f)$ by introducing a subgroup $G$ of $\mathrm{GL}(V)$ and applying the theory of semisimple algebraic groups. Along the way, we give an explicit description of the normalizer $N_{\mathrm{GL}(V)}(G)$ and prove that, under a mild technical assumption, the normalizer agrees with the stabilizer in $\mathrm{GL}(V)$ of the orbit of the highest weight vector in $\mathbb{P}(V)$.
\end{abstract}

In an 1897 paper [Fro97, Frobenius proved that every linear transformation of the $n$-by- $n$ real matrices that preserves the determinant is of the form

$$
X \mapsto A X B \quad \text { or } \quad X \mapsto A X^{t} B
$$

for some $A, B \in \mathrm{GL}_{n}(\mathbb{R})$ such that $\operatorname{det}(A B)=1$; that is, the obvious ones are the only ones. This is the basic example of a solution to a linear preserver problem (LPP): one is given a finite-dimensional vector space $V$ over a field $K$ and a polynomial function $f: V \rightarrow K$ and one wants to determine the linear transformations of $V$ that preserve $f$. Since Frobenius, many such problems have been solved; see

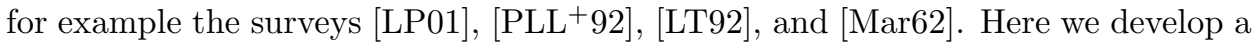
general method that solves several new problems; see Examples 3.15 and 3.16 and Corollaries 8.11, 9.3, 9.5, 9.7, 9.8, and 9.11.

Our method is to introduce an auxiliary group $G \subset \mathrm{GL}(V)$ that is semisimple and such that $V$ is an irreducible representation or Weyl module of $G$. In 2 , we determine the normalizer $N_{\mathrm{GL}(V)}(G)$ of $G$ in $\mathrm{GL}(V)$. We prove in Theorem 3.2 that this subgroup equals the stabilizer of a closed $G$-orbit $\mathscr{O}$ in the projective space $\mathbb{P}(V)$ - i.e., $\operatorname{Stab}_{\mathrm{GL}(V)}(\mathscr{O})=N_{\mathrm{GL}(V)}(G)$ - under a mild technical assumption on the isotropy subgroup. Using this result, in $\S 98$ and 9 we solve two families of LPPs by reducing the problems in each family to determining this stabilizer. These two families consist of representations $V$ with a 1-dimensional ring of $G$-invariant functions generated by $f$ and are examples of prehomogeneous vector spaces of parabolic type. The two families correspond to the cases where the unipotent radical $U$ of the parabolic subgroup is abelian (i.e., $[U, U]=0$ ) or $[U, U]$ is 1 dimensional respectively, and we use the general results on representations in these families from RRS92, Röh93], and [Hel12].

Besides obtaining new results, we also recover many known solutions to linear preserver problems. The generality of our method is in contrast to many of the proofs in the literature, which typically are highly dependent on the particular choice of $V$ and $f$. (The arguments in [PD95] and Gur97] are notable exceptions.)

Received by the editors January 24, 2012 and, in revised form, October 20, 2012.

2010 Mathematics Subject Classification. Primary 47B49; Secondary 15A04, 15A72, 20 G15.

(C)2014 American Mathematical Society Reverts to public domain 28 years from publication 
Further, we require only very weak assumptions about the field $K$ (at most we require that $K$ is infinite or has characteristic $\neq 2,3$ ) and determine the preserver precisely (and not just its identity component or Lie algebra).

Applications of solutions to LPPs. Linear preserver problems arise naturally in algebra, sometimes in nonobvious ways. For example, every associative division algebra $D$ that is finite-dimensional over its center $K$ has a "generic characteristic polynomial" generalizing the notion of a characteristic polynomial on $n$-by- $n$ matrices. Its coefficients are polynomial functions $E_{r}: D \rightarrow K$ for $1 \leq r \leq \sqrt{\operatorname{dim}_{K} D}$ where $E_{r}$ has degree $r$. By determining the preserver of $E_{r}$, Waterhouse proved that $D$ is determined up to isomorphism or anti-isomorphism by $E_{r}$ for any $r \geq 3$; see Wat82 or [Wat95, Cor. 4]. This in turn gives a result on the essential dimension of central simple algebras; see [Flo11.

Notation. An affine group scheme $G$ over a field $K$ is a representable functor from commutative $K$-algebras to the category of groups, i.e., it is given by $S \mapsto$ $\operatorname{Hom}_{K \text {-alg. }}(K[G], S)$ for some $K$-algebra $K[G]$ and every $K$-algebra $S$; see [Wat79]. We are mostly concerned with the case where $G$ is a (linear) algebraic group, i.e., where $K[G]$ is smooth of finite type over $K$. The "concrete" group $G(S)$ is called the group of $S$-points. We maintain the distinction between $G$ and $G(S)$ except for the group $\mathrm{GL}(V)$ of linear transformations on a finite-dimensional $K$-vector space $V$. For that group context will show whether the group scheme or the collection of invertible $K$-linear transformations on $V$ ("GL( $V)(K)$ ") is meant.

For (Zariski) closed subgroups $G, N$ of GL( $V)$, we write $G . N$ for the compositum of $G$ and $N$, i.e., for the smallest closed subgroup of $\operatorname{GL}(V)$ containing $G$ and $N$. If $N$ normalizes $G$ and $K$ is algebraically closed, $(G . N)(K)=G(K) \cdot N(K)$.

Throughout we use notions from the theory of semisimple groups as in DG70, [Bor91, Hum81], Hum80, or [Bou05], such as roots and weights.

\section{IRREDUCIBLE REPRESENTATIONS AND THE CLOSED ORBIT}

We now describe the basic setup that will be used throughout the paper, providing details and examples for the convenience of the reader who is a nonspecialist in semisimple groups.

Let $\widetilde{G}$ be a split semisimple linear algebraic group over a field $K$ and fix a representation $\rho: \widetilde{G} \rightarrow \mathrm{GL}(V)$-our definition of semisimple includes the fact that $\widetilde{G}$ is connected. Table $\mathrm{A}$ lists some examples of pairs $(\widetilde{G}, V)$ that we will consider. For notational simplicity, we focus on the image $G$ of $\widetilde{G}$ in $\operatorname{GL}(V)$. This group is also split semisimple. We will assume that $V$ is an irreducible representation or is a Weyl module in the sense of [Jan03, p. 183]. (If char $K=0$, the two notions coincide.) In either case, $\operatorname{End}_{G}(V)=K$; see loc. cit. when $V$ is a Weyl module.

Fix a pinning of $G$ in the sense of [DG70, §XXIII.1] (called a "framing" in Bou05]); this includes choosing a split maximal $K$-torus $T$, a set of simple roots $\Delta$ of $G$ with respect to $T$, and a corresponding Borel subgroup $B$. Recall that $T^{*}$ is naturally included in the weight lattice and there are bijections between dominant weights in $T^{*}$, equivalence classes of irreducible representations of $G$, and equivalence classes of Weyl modules of $G$ [Jan03, II.2.4]. Put $\lambda \in T^{*}$ for the highest weight of $V$ and $v^{+}$for a highest weight vector in $V$. 
The stabilizer of $K v^{+}$in $G$ contains the Borel subgroup $B$, so it is a parabolic subgroup $P$. The orbit $\mathscr{O}$ of $K v^{+}$is identified with the projective variety $G / P$, so $\mathscr{O}$ is closed in $\mathbb{P}(V)$.

Definition 1.1. We call an element $x \in V$ minimal if $K x$ belongs to $\mathscr{O}$.

Example 1.2 (Exterior powers). Take $\widetilde{G}=\mathrm{SL}_{n}$ and $V=\bigwedge^{d}\left(K^{n}\right)$ for some $d$ between 1 and $n$. The group acts via $\rho(g)\left(v_{1} \wedge v_{2} \wedge \cdots \wedge v_{d}\right)=g v_{1} \wedge g v_{2} \wedge \cdots \wedge g v_{d}$ for $v_{1}, \ldots, v_{d} \in K^{n}$. The image $G$ of $\widetilde{G}$ is equal to $\mathrm{SL}_{n} / \mu_{e}$, where $\mu_{e}$ is the group scheme of $e$-th roots of unity for $e:=\operatorname{gcd}(d, n)$. (Recall that, as a scheme, $\mu_{e}$ is Spec $K[x] /\left(x^{e}-1\right)$, so it is smooth if and only if char $K$ does not divide $e$.) For $T$ and $B$, we take the image in $G$ of the diagonal and upper-triangular matrices, respectively. The only line stabilized by $B$ is the span of $v^{+}=e_{1} \wedge \cdots \wedge e_{d}$, where $e_{i}$ denotes the element of $K^{n}$ with a 1 in the $i$-th position and zeros elsewhere.

The group $\widetilde{G}$ is of type $A_{n-1}$ and its Dynkin diagram $\Delta$ is

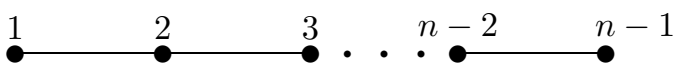

where we have labeled each vertex with the number $i$ of the corresponding fundamental weight $\omega_{i}$ according to the numbering from Bou02. With respect to this numbering, $v^{+}$has weight $\lambda=\omega_{d}$. The representation $V$ is irreducible because $\omega_{d}$ is minuscule.

An element of $V$ is decomposable if it can be written as $v_{1} \wedge \cdots \wedge v_{d}$ for some $v_{i} \in K^{n}$. As $\mathrm{SL}_{n}$ acts transitively on the $d$-dimensional subspaces of $K^{n}$, we conclude that the minimal elements in $V$ are the nonzero decomposable vectors.

In the special case $d=2$, we may identify $V$ with the vector space $\mathrm{Skew}_{n}$ of $n$-by$n$ alternating matrices - i.e., skew-symmetric matrices with zeros on the diagonal (the extra condition is necessary if char $K=2$ ) - where $\mathrm{SL}_{n}$ acts via $\rho(g) v=g v g^{t}$. Then $v^{+}$corresponds to $E_{12}-E_{21}$, where $E_{i j}$ denotes a matrix with a 1 in the $(i, j)$-entry and zeros elsewhere. From this, we see that the minimal elements are the alternating matrices of rank 2.

Example 1.3 ("Symmetric powers"). Take $\widetilde{G}=\mathrm{SL}_{n}$ (with fundamental weights numbered as in the previous example) and take $V$ to be the Weyl module with highest weight $\lambda:=d \omega_{1}$ for some $d \geq 1$. The group $G$ is $\mathrm{SL}_{n} / \mu_{e}$ as in the previous example. If char $K$ is zero or $>d$, this representation is irreducible by, for example, [Gre80, p. 50], and in that case $V$ can be identified with the $d$-th symmetric power $S^{d}\left(K^{n}\right)$ of the tautological representation, the highest weight line is spanned by $v^{+}=e_{1}^{d}$, and minimal elements are the $d$-th powers of nonzero elements of $K^{n}$.

When $d=2$ and char $K \neq 2$, we may identify $V$ with the vector space $\operatorname{Symm}_{n}$ of $n$-by- $n$ symmetric matrices, where $\mathrm{SL}_{n}$ acts by $\rho(g) v=g v g^{t}, v^{+}$corresponds to $E_{11}$, and the minimal elements are symmetric matrices of rank 1.

Returning to the case of general $G$ and $V$, we have:

Example 1.4. The collection of minimal elements is nonempty and $G(K)$-invariant, so it spans a $G(K)$-invariant subspace of $V$ that contains $v^{+}$; hence it must be all of $V$. Therefore, there is a basis of $V$ consisting of minimal elements. 
Said differently, the set $\{x \in V \mid K x \in \mathscr{O}(K)\}$ spans $V$. This property characterizes $\mathscr{O}$, regardless of $K$ :

Lemma 1.5. If $\mathscr{O}^{\prime}$ is a closed $G$-orbit in $\mathbb{P}(V)$ and the set $\left\{x \in V \mid K x \in \mathscr{O}^{\prime}(K)\right\}$ spans $V$, then $\mathscr{O}^{\prime}=\mathscr{O}$.

Proof. For $K_{\text {alg }}$ an algebraic closure of $K$, the set $\left\{x \in V \otimes K_{\text {alg }} \mid K_{\text {alg }} x \in \mathscr{O}^{\prime}\left(K_{\text {alg }}\right)\right\}$ spans $V \otimes K_{\text {alg. }}$. As it suffices to verify $\mathscr{O}=\mathscr{O}^{\prime}$ over $K_{\text {alg }}$, we may assume that $K$ is algebraically closed.

By the Borel Fixed Point Theorem, there is a line $K x \in \mathscr{O}^{\prime}(K)$ that is stabilized by $B$. Hence $x$ is a weight vector for some $\mu \in T^{*}$, which is dominant because $\operatorname{Hom}_{G}(V(\mu), V)$ is nonzero [Jan03, p. 183, Lemma 2.13(a)]. All the weights of the $G$-submodule of $V$ generated by $x$ are $\leq \mu$ by loc. cit., but this submodule is all of $V$, so $\mu$ must equal $\lambda$ and $K v^{+}=K x$.

When $V$ is irreducible, the spanning condition is not necessary, because the set $\left\{x \in V \mid K x \in \mathscr{O}^{\prime}(K)\right\}$ spans $V$ for every orbit $\mathscr{O}^{\prime}$ with $\mathscr{O}^{\prime}(K)$ nonempty; hence the well-known result: $\mathscr{O}$ is the unique closed $G$-orbit in $\mathbb{P}(V)$.

When $V$ is a Weyl module, the spanning condition is necessary. Indeed, if there is an exact sequence of representations $1 \rightarrow A \rightarrow V \rightarrow B \rightarrow 1$ where $A$ and $B$ are irreducible, then the closed orbit in $\mathbb{P}(A)$ gives a closed orbit in $\mathbb{P}(V)$ distinct from $\mathscr{O}$. This occurs, for example, when $G=\mathrm{SL}_{2}$ over a field $K$ of characteristic 3 and $V$ is the Weyl module with highest weight 3 , in which case we additionally have that the two closed orbits are isomorphic as varieties (to $\mathbb{P}^{1}$ ).

It is harmless to identify $\mathbb{G}_{m}$ — the algebraic group with $S$-points $S^{\times}$— with the subvariety of scalar matrices in $\mathrm{GL}(V)$.

Corollary 1.6. The sub-group-scheme of $\mathrm{GL}(V)$ fixing $\mathscr{O}$ elementwise is the group $\mathbb{G}_{m}$ of scalar matrices. In particular, it is smooth.

Proof. Put $H$ for the sub-group-scheme fixing $\mathscr{O}$. For each $h \in H(K)$, there is a morphism $\mathscr{O} \rightarrow \mathbb{G}_{m}$ defined by $[v] \mapsto h v / v$. As $\mathscr{O}$ is projective connected and $\mathbb{G}_{m}$ is affine, the image must be a point. That is, there is a $c \in K^{\times}$such that $h v=c v$ for every minimal $v \in V$. Example 1.4 gives the fact that $h$ is a scalar matrix.

A similar argument shows that $H$ is smooth. Put $K[\varepsilon]$ for the dual numbers (with $\varepsilon^{2}=0$ ) and suppose $h=1+x \varepsilon$ is in $H(K[\varepsilon]) ; x$ can naturally be viewed as a (possibly noninvertible) linear transformation of $V$. The equation $(1+x \varepsilon) v=$ $v+\varepsilon \lambda_{v} v$ defines a morphism $\mathscr{O} \rightarrow \mathbb{A}^{1}$ via $[v] \mapsto \lambda_{v}$. This map must be constant; therefore $x$ is the scalar $\lambda_{v}$ and $\operatorname{dim} \operatorname{Lie}(H)=1$, i.e., $H$ is smooth. From this and equality of $K_{\text {alg-points }} \mathbb{G}_{m}\left(K_{\text {alg }}\right)=H\left(K_{\text {alg }}\right)$, we deduce that $\mathbb{G}_{m}=H$.

Corollary 1.7. If $K$ is infinite, then the subgroup of $\mathrm{GL}(V)(K)$ fixing $\mathscr{O}(K)$ elementwise consists of the scalar matrices.

Proof. Let $g \in \mathrm{GL}(V)(K)$ fix $\mathscr{O}(K)$. Then, as $\mathscr{O}$ is a rational variety Bor91, 21.20(ii)] and $K$ is infinite, $\mathscr{O}(K)$ is dense in $\mathscr{O}$ and the automorphism of $\mathbb{P}(V)$ induced by $g$ fixes $\mathscr{O}$ as a variety. Corollary 1.6 gives the fact that $g$ is a scalar matrix.

For analogous results proved in various special cases, see for example the end of Die49, Prop. 8 in Jac61, or Cor. 6.3 in [Fer72.

Section 3 below is concerned with calculating the stabilizer $\operatorname{Stab}_{\mathrm{GL}(V)}(\mathscr{O})$ of $\mathscr{O}$ in $\mathrm{GL}(V)$, which is a group scheme whose $K$-points are the elements of $\mathrm{GL}(V)(K)$ 
that normalize $\mathscr{O}(R)$ for every commutative $K$-algebra $R$. We make some general remarks about it here.

Lemma 1.8. If $K$ is infinite and $T \in \mathrm{GL}(V)(K)$ stabilizes $\mathscr{O}(K)$, then $T$ is a $K$-point of $\operatorname{Stab}_{\mathrm{GL}(V)}(\mathscr{O})$.

Proof. Identical to the proof of Corollary 1.7.

In the literature on linear preserver problems, authors are sometimes concerned with calculating all matrices that stabilize $\mathscr{O}$ and not just the invertible ones. Note that a linear transformation $T$ may preserve $\mathscr{O}(K)$ but fail to be invertible because $(\operatorname{ker} T) \cap \mathscr{O}$ may have no $K$-points. This happens even in the very nice case where $k=\mathbb{R}$; see e.g. Zha05, Example 1]. However, it is sufficient to check that $T$ stabilizes the collection of minimal elements in $V \otimes K_{\text {alg: }}$ :

Proposition 1.9. If a linear transformation $T$ of $V$ stabilizes $\mathscr{O}\left(K_{\mathrm{alg}}\right)$, then $T$ is invertible and is a $K$-point of $\operatorname{Stab}_{\mathrm{GL}(V)}(\mathscr{O})$.

Proof. We adapt the argument from [CL92, p. 322]. The set $X$ of minimal elements in $V$ has closure $\bar{X}=X \cup\{0\}$, an irreducible subvariety of $V$. Note that $T(\bar{X})$ is a closed subvariety of $\bar{X}$, as can be seen by considering the morphism induced by $T$ on the image $\mathscr{O}$ of $X$ in $\mathbb{P}(V)$. The fiber of $T: \bar{X} \rightarrow T(\bar{X})$ over 0 is just $\{0\}$, and we deduce that $\operatorname{dim} T(\bar{X})=\operatorname{dim} \bar{X}[$ Hum81, Th. 4.1]; hence $T(\bar{X})=\bar{X}$. As $\bar{X}(K)$ contains a spanning set for $V$ (Example 1.4) $T$ is invertible. Lemma 1.8 shows that $T$ is contained in $\operatorname{GL}(V)(K) \cap \operatorname{Stab}_{\mathrm{GL}(V)}(\mathscr{O})\left(K_{\mathrm{alg}}\right)$, i.e., $\operatorname{Stab}_{\mathrm{GL}(V)}(\mathscr{O})(K)$.

In older times, this was proved by hand for each choice of $G$ and $V$; see for example [Wes69.

\section{The nORMALIZER OF $G$ IN $\operatorname{GL}(V)$}

The purpose of this section is to precisely describe the structure of the normalizer of $G$ in $\operatorname{GL}(V)$, Proposition 2.2 below. We maintain the notation and hypotheses of $\$ 1$.

Write $\operatorname{Aut}(\Delta)$ for the automorphism group of the Dynkin diagram of $G$. (This is an abuse of notation in that we have already defined $\Delta$ to be the set of simple roots, i.e., the vertex set of the Dynkin diagram.) We write $\operatorname{Aut}(\Delta, \lambda)$ for the subgroup of $\operatorname{Aut}(\Delta)$ fixing $\lambda$.

Example 2.1. Returning to Examples 1.2 and 1.3, the group $\operatorname{Aut}(\Delta)$ acts on the weights by permuting the fundamental weights according to its action on the diagram. We find that $\operatorname{Aut}(\Delta, \lambda)=1$ for $V$ with highest weight $d \omega_{1}$, but for $V=\bigwedge^{d}\left(K^{n}\right)$ we have $\operatorname{Aut}(\Delta, \lambda)=1$ for $n \neq 2 d$, and $\mathbb{Z} / 2 \mathbb{Z}$ for $n=2 d$ (in particular, for $\mathrm{SL}_{4}$ acting on the 4-by-4 alternating matrices).

Write $\operatorname{Aut}(G, \lambda)$ for the inverse image of $\operatorname{Aut}(\Delta, \lambda)$ under the map $\operatorname{Aut}(G) \rightarrow$ $\operatorname{Aut}(\Delta)$. To spell this out, recall that given split maximal $K$-tori $T_{1}, T_{2}$ and Borel $K$ subgroups $B_{1}, B_{2}$ in $G$ such that $T_{i} \subset B_{i}$, there is a $g \in G(K)$ so that $g T_{1} g^{-1}=T_{2}$ and $g B_{1} g^{-1}=B_{2}$ [Bor91, 19.2, 20.9(i)]. Therefore, given an automorphism $\phi$ of $G$, we may compose it with conjugation by an element of $G(K)$ to produce an element $\phi^{\prime}$ such that $\phi^{\prime}(T)=T$ and $\phi^{\prime}(B)=B$. The automorphism $\phi^{\prime}$ is determined up to conjugation by an element of $T$, so the action of $\phi^{\prime}$ on $T^{*}$ is uniquely determined by $\phi$. Then $\operatorname{Aut}(G, \lambda)(K)$ is the collection of $\phi \in \operatorname{Aut}(G)(K)$ such that $\phi^{\prime}(\lambda)=\lambda$. 
The pinning induces a homomorphism $i$ embedding $\operatorname{Aut}(\Delta)$ in the automorphism group of the simply connected cover of $G$ [DG70, XXIII.4, Th. 4.1]. Further, writing $Z$ for the center of $G$, we have:

Proposition 2.2. The map $i$ induces a homomorphism $\operatorname{Aut}(\Delta, \lambda) \rightarrow \operatorname{Aut}(G, \lambda)$ and an injection $\gamma$ such that the diagram

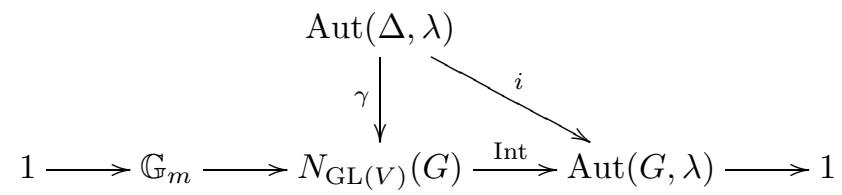

commutes, the horizontal sequence is exact, and Int is surjective on $K$-points. Furthermore, $N_{\mathrm{GL}(V)}(G)$ is smooth and $\gamma$ identifies $N_{\mathrm{GL}(V)}(G)$ with $\left(\left(\mathbb{G}_{m} \times G\right) / Z\right) \rtimes$ $\operatorname{Aut}(\Delta, \lambda)$.

In the statement, we wrote Int for the map such that $\operatorname{Int}(n)(g)=n g n^{-1}$ for $n \in N_{\mathrm{GL}(V)}(G)(R)$ and $g \in G(R)$, for every $K$-algebra $R$. For a definition of short exact sequences of affine group schemes and their basic properties, see for example [KMRT98, p. 341].

Proof. For the purpose of this proof, write $\widetilde{G}$ for the simply connected cover of $G$ and $\widetilde{Z}$ for its center. For $\pi \in \operatorname{Aut}(\Delta, \lambda), i(\pi) \in \operatorname{Aut}(\widetilde{G})$ normalizes $\left.\operatorname{ker} \lambda\right|_{\widetilde{Z}}$, which is the kernel of $\widetilde{G} \rightarrow G$. Hence $i(\pi)$ induces an automorphism of $G$. As $i$ is a section of the natural homomorphism $\operatorname{Aut}(\widetilde{G}) \rightarrow \operatorname{Aut}(\Delta)$, it is also a section of the natural homomorphism $\operatorname{Aut}(G, \lambda) \rightarrow \operatorname{Aut}(\Delta, \lambda)$. It follows from this discussion that $i$ identifies $\operatorname{Aut}(G, \lambda)$ with $(G / Z) \rtimes \operatorname{Aut}(\Delta, \lambda)$.

We claim that $(G / Z)(K)$ is in the image of $N_{G L(V)}(G)(K)$. Indeed, the normalizer contains $G$ and the scalar matrices $\mathbb{G}_{m}$, and these two groups have intersection $Z$. This gives an exact sequence

$$
1 \longrightarrow \mathbb{G}_{m} \longrightarrow\left(\mathbb{G}_{m} \times G\right) / Z \stackrel{\text { Int }}{\longrightarrow} G / Z \longrightarrow 1 .
$$

Applying Galois cohomology gives an exact sequence

$$
\left(\left(\mathbb{G}_{m} \times G\right) / Z\right)(K) \stackrel{\text { Int }}{\longrightarrow}(G / Z)(K) \longrightarrow H^{1}\left(K, \mathbb{G}_{m}\right),
$$

where the last term is zero by Hilbert's Theorem 90 [KMRT98, 29.3]. As the first term is contained in $N_{\mathrm{GL}(V)}(G)(K)$, we have verified the claim.

For $n \in N_{\mathrm{GL}(V)}(G)(K)$, $\operatorname{Int}(n)$ is an automorphism of $G$, and modifying it by conjugation by an element of $G(K)$, we may assume that $\operatorname{Int}(n)$ normalizes $B$ and $T$. As Int $(n)$ defines an equivalence of the irreducible representations or Weyl modules with highest weights $\lambda$ and ${ }^{n} \lambda$, we deduce that $\operatorname{Int}(n)$ belongs to $\operatorname{Aut}(G, \lambda)(K)$. Running this argument backwards shows that Int is surjective on $K$-points. This also completes the proof that the sequence is exact and that $N_{\mathrm{GL}(V)}(G)$ is smooth (because $\mathbb{G}_{m}$ and $\operatorname{Aut}(G, \lambda)$ are KMRT98, 22.12]).

To construct $\gamma$, we take $\pi \in \operatorname{Aut}(\Delta, \lambda)(K)$. The element $n$ such that $\operatorname{Int}(n)=$ $i(\pi)$ is determined up to a factor in $K^{\times}$; we pick $n$ so that $n v^{+}=v^{+}$and put $\gamma(\pi):=$ $n$. To verify that it is a homomorphism, note that $\operatorname{Int}\left(\gamma\left(\pi_{1} \pi_{2}\right)\right)=\operatorname{Int}\left(\gamma\left(\pi_{1}\right) \gamma\left(\pi_{2}\right)\right)$, so $\gamma\left(\pi_{1} \pi_{2}\right)$ and $\gamma\left(\pi_{1}\right) \gamma\left(\pi_{2}\right)$ differ by at most a factor in $K^{\times}$. But both elements of $\mathrm{GL}(V)$ fix $v^{+}$, so they are equal. 
For the final claim, note that if $\pi \in \operatorname{Aut}(\Delta, \lambda)$ is such that $\gamma(\pi)$ is in the identity component of $N_{\mathrm{GL}(V)}(G)$, then Int $\gamma(\pi)=i(\pi)$ belongs to the identity component of $\operatorname{Aut}(G, \lambda)$, i.e., to $G / Z$, and we conclude that the semidirect product $N^{\prime}$ of $N_{\mathrm{GL}(V)}(G)^{\circ}$ and $\gamma(\operatorname{Aut}(\Delta, \lambda))$ is identified with a subgroup of the normalizer. Furthermore, writing $\pi_{0}$ to mean the component group, we have

$$
\gamma(\operatorname{Aut}(\Delta, \lambda))=\pi_{0}\left(N^{\prime}\right) \subseteq \pi_{0}\left(N_{\mathrm{GL}(V)}(G)\right)=\pi_{0}(\operatorname{Aut}(G, \lambda))=i(\operatorname{Aut}(\Delta, \lambda)),
$$

so $N^{\prime}$ equals $N_{\mathrm{GL}(V)}(G)$. Exactness of (2.3) completes the proof.

Corollary 2.4. If $K$ is algebraically closed, then $N_{\mathrm{GL}(V)}(G)(K)$ is generated by $G(K), K^{\times}$, and $\gamma(\operatorname{Aut}(\Delta, \lambda))$.

In many of the examples considered below, the following holds:

There is a connected reductive group $\widetilde{L}$ such that $\widetilde{G}$ is the derived group of $\widetilde{L}$ and $\rho$ extends to a homomorphism $\widetilde{\rho}: \widetilde{L} \rightarrow \mathrm{GL}(V)$ such that im $\widetilde{\rho}$ contains the scalar matrices and $\operatorname{ker} \widetilde{\rho}$ is a split torus.

This allows us to make the more attractive statement, which holds with no hypotheses on $K$ :

Corollary 2.6. Assuming (2.5), $N_{\mathrm{GL}(V)}(G)(K)$ is the subgroup of $\mathrm{GL}(V)$ generated by $\widetilde{\rho}(\widetilde{L}(K))$ and $\gamma(\operatorname{Aut}(\Delta, \lambda))$.

Proof. The sequence $1 \rightarrow \operatorname{ker} \widetilde{\rho} \rightarrow \widetilde{L} \stackrel{\widetilde{\rho}}{\rightarrow} N_{\mathrm{GL}(V)}(G)^{\circ} \rightarrow 1$ is exact by the preceding corollary, so $\widetilde{L}(K) \rightarrow N_{\mathrm{GL}(V)}(G)^{\circ}(K) \rightarrow H^{1}(K, \operatorname{ker} \widetilde{\rho})$ is exact. But the last term is 1 by Hilbert 90 because $\operatorname{ker} \widetilde{\rho}$ is a split torus.

\section{LinEAR TRANSFORMATIONS PRESERVING MINIMAL ELEMENTS}

We maintain the notation and hypotheses of $\$ 1$. We will determine the stabilizer $\operatorname{Stab}_{\mathrm{GL}(V)}(\mathscr{O})$ of $\mathscr{O}$ in $\mathrm{GL}(V)$ as an affine group scheme.

Example 3.1. If $n \in \mathrm{GL}(V)$ normalizes $G$, then for every minimal $x$, the $G$-orbit of $n x$ in $\mathbb{P}(V)$ is closed and spans $V$, hence $n x$ is also minimal by Lemma 1.5. That is, $N_{\mathrm{GL}(V)}(G)(K)$ is contained in $\operatorname{Stab}_{\mathrm{GL}(V)}(\mathscr{O})(K)$.

Under a technical hypothesis spelled out in Definition 3.3 , we can say that this containment is an equality. Recall that $P$ is the parabolic subgroup of $G$ stabilizing the highest weight line $K v^{+}$.

Theorem 3.2. $\operatorname{Stab}_{\mathrm{GL}(V)}(\mathscr{O})=N_{\mathrm{GL}(V)}(G)$ if $P$ is not exceptional.

We delay the proof temporarily.

Definition 3.3. Following Dem77, we define:

(1) If $G$ is simple, then $P$ is exceptional in the following cases:

(a) $G$ has type $C_{\ell}$ with $\ell \geq 2$ and $P$ has Levi subgroup of type $C_{\ell-1}$.

(b) $G$ has type $B_{\ell}$ with $\ell \geq 2$ and $P$ has Levi subgroup of type $A_{\ell-1}$.

(c) $G$ has type $G_{2}$ and $P$ is the stabilizer of the highest weight vector in the 7-dimensional fundamental Weyl module.

(2) If $G$ is not simple, we write its adjoint group as $\bar{G}_{1} \times \cdots \times \bar{G}_{r}$ where each $\bar{G}_{i}$ is simple. We say that $P$ is exceptional if at least one of its images in $\bar{G}_{1}, \ldots, \bar{G}_{r}$ is exceptional. 
For each of the representations listed in Table $\mathrm{A}, P$ is not exceptional, so in these cases Theorem 3.2 applies and $N_{\mathrm{GL}(V)}(G)=\operatorname{Stab}_{\mathrm{GL}(V)}(\mathscr{O})$.

All three cases in Definition 3.3(1) give genuine exceptions to Theorem 3.2, Item (1a) includes the case where $G=\mathrm{Sp}_{2 \ell}$ for $\ell \geq 2$ and $V$ is the natural representation. In that case, every nonzero vector is a minimal element, so $\operatorname{Stab}_{\mathrm{GL}_{2 \ell}}(\mathscr{O})$ is all of $\mathrm{GL}_{2 \ell}$, but $N_{\mathrm{GL}_{2 \ell}}\left(\mathrm{Sp}_{2 \ell}\right)$ is $\mathbb{G}_{m} . \mathrm{Sp}_{2 \ell}$, which has dimension only $2 \ell^{2}+\ell+1$. Item $(1 \mathrm{~b})$ is addressed in 10 . Item (1c) includes the case where $G$ is the automorphism group of the split octonions and $V$ is the space of trace zero octonions. In that case, the closed $G$-orbit is the quadric in $\mathbb{P}(V)$ defined by the quadratic norm form $q$ [CG06, 9.2]; hence $\operatorname{Stab}_{\mathrm{GL}(V)}(\mathscr{O})$ is the group of similarities of $q$. This has dimension 22 as opposed to $\operatorname{dim} \mathbb{G}_{m} \cdot G=15$.

Remark. Demazure includes a fourth item in his version of Definition 3.3, namely that $G \neq 1$ and $P=G$, which would appear as (1d) in Definition 3.3 above. But this case cannot occur here due to our assumption that the representation $V$ is faithful.

Proof of Theorem 3.2. We abbreviate $N:=N_{\mathrm{GL}(V)}(G)$ and $S:=\operatorname{Stab}_{\mathrm{GL}(V)}(\mathscr{O})$. Consider the diagram

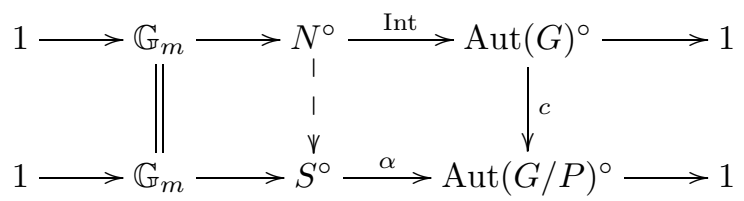

where $c$ is given by

$$
c(\operatorname{Int}(x)) g P=x g P \quad \text { for } g \in G(K) \text { and } \operatorname{Int}(x) \in \operatorname{Aut}(G)^{\circ}(K) .
$$

The top sequence is exact by Proposition 2.2. As $P$ is not exceptional, Dem77, Th. 1] gives that $c$ is an isomorphism, hence $\alpha$ is surjective. From this and Corollary 1.6 we see that the bottom sequence is exact and, in particular, $S^{\circ}$ is smooth (because $\operatorname{Aut}(G / P)^{\circ}$ and $\mathbb{G}_{m}$ are smooth). Because $N^{\circ}$ is also smooth, the inclusion $N^{\circ}(K) \subseteq S^{\circ}(K)$ given by Example 3.1 provides an inclusion of algebraic groups, represented by the dashed arrow in (3.4). The inequalities

$$
1+\operatorname{dim} \operatorname{Aut}(G)^{\circ}=\operatorname{dim} N^{\circ} \leq \operatorname{dim} S^{\circ}=1+\operatorname{dim} \operatorname{Aut}(G)^{\circ}
$$

give that $S^{\circ}=N^{\circ}$.

Recall from Proposition 2.2 that $N^{\circ}$ is reductive with semisimple part $G$; hence $G$ is a characteristic subgroup of $S^{\circ}$. As $S$ normalizes $S^{\circ}$, we deduce that $S$ normalizes $G$.

We now describe the matrices in $\operatorname{GL}(V)$ that stabilize $\mathscr{O}(K)$ for various interesting choices of $V$ and $\mathscr{O}$. We rely on Lemma 1.8 for the fact that this stabilizer equals the (a priori smaller) group $\operatorname{Stab}_{\mathrm{GL}(V)}(\mathscr{O})(K)$ described by Theorem 3.2 and Proposition 2.2. so we assume $K$ is infinite. However, this hypothesis is not necessary and can be avoided using an argument from finite group theory; see the references preceding each statement for a special argument in each case.

For the following result compare [Lim79, Cor. 2], Wat87, Th. 6.5], Wat89, Th. 11], or Gur97, Cor. 6.2]. 
Corollary 3.5 (Symmetric matrices). Suppose $K$ is infinite and has characteristic $\neq 2$. Every invertible linear transformation of $\operatorname{Symm}_{n}(K)$ that sends rank 1 matrices to rank 1 matrices is of the form

$$
X \mapsto r P X P^{t} \quad \text { for some } r \in K^{\times} \text {and } P \in \mathrm{GL}_{n}(K) \text {. }
$$

Proof. Take $\widetilde{G}=\mathrm{GL}_{n}$ as in Example 1.3. In the notation of (2.5), take $\widetilde{L}=$ $\mathbb{G}_{m} \times \mathrm{GL}_{n}$, and for $(r, P) \in K^{\times} \times \mathrm{GL}_{n}(K)$, define $\widetilde{\rho}(r, P)$ as in (3.6). For every commutative $K$-algebra $R$, the set of $R$-points of $\operatorname{ker} \widetilde{\rho}$ is $\left\{\left(t^{2}, t^{-1}\right) \mid t \in R^{\times}\right\}$. As $\operatorname{Aut}(\Delta, \lambda)=1$, combining Corollary 2.6 and Theorem 3.2 gives the claim.

For the next result compare [MW60, Th. 3], Wat87, Th. 5.5], or Gur97, Cor. 7.3].

Corollary 3.7 (Alternating matrices). Suppose $K$ is infinite. For $n \geq 2$ and $n \neq$ 4, every invertible linear transformation of $\operatorname{Skew}_{n}(K)$ that sends rank 2 matrices to rank 2 matrices is of the form (3.6). If $n=4$, then every invertible linear transformation of $\operatorname{Skew}_{n}(K)$ that sends rank 2 matrices to rank 2 matrices is as in (3.6) or is

$$
X \mapsto r P X^{*} P^{t} \quad \text { for some } r \in K^{\times}, P \in \mathrm{GL}_{n}(K)
$$

and

$$
\left(\begin{array}{cccc}
0 & x_{1} & x_{2} & x_{3} \\
-x_{1} & 0 & x_{4} & x_{5} \\
-x_{2} & -x_{4} & 0 & x_{6} \\
-x_{3} & -x_{5} & -x_{6} & 0
\end{array}\right)^{*}=\left(\begin{array}{cccc}
0 & x_{1} & -x_{2} & -x_{4} \\
-x_{1} & 0 & -x_{3} & -x_{5} \\
x_{2} & x_{3} & 0 & x_{6} \\
x_{4} & x_{5} & -x_{6} & 0
\end{array}\right) .
$$

The map $*$ is a Hodge star operator, which is not uniquely determined. Said differently, one can replace $*$ with its composition by any map as in (3.6). Therefore, one finds slightly different formulas in other sources, such as [MW60, p. 921] and $\left[\mathrm{PLL}^{+} 92\right.$, p. 15].

Proof of Corollary 3.7 . We use the same $\widetilde{L}$ and $\widetilde{\rho}$ from the proof of the preceding corollary, substituting $\operatorname{Skew}_{n}(K)$ for $V$. If $n \neq 4$, Aut $(\Delta, \lambda)=1$, and the proof is complete. Otherwise $n=4$, Aut $(\Delta, \lambda)=\mathbb{Z} / 2 \mathbb{Z}$, and we are tasked with finding the image of the nonidentity element $\pi$ of $\operatorname{Aut}(\Delta, \lambda)$ under the map $\gamma$ from Proposition 2.2. The element $*$ of GL $(V)$ fixes $v^{+}=E_{12}-E_{21}$. Furthermore, one checks that $\operatorname{Int}(*)$ normalizes the maximal torus $T$ and permutes the root subgroups (described in [Bou05, §VIII.13.3]) as indicated by the action of $\pi$ on $\Delta$; it follows that * normalizes $G$, hence $\gamma(\pi)=*$.

For the next result compare Hua48, [MM59b, Th. 1], Min77, Th. 1], or Wat87. Th. 3.5].

Corollary 3.10 (Rectangular matrices). Suppose $K$ is infinite. For $m, n \geq 2$ and $m \neq n$, every invertible linear transformation of the $m$-by-n matrices with entries in $K$ that sends rank 1 matrices to rank 1 matrices is of the form

$$
X \mapsto A X B \text { for some } A \in \mathrm{GL}_{m}(K) \text { and } B \in \mathrm{GL}_{n}(K) \text {. }
$$

For $n=m \geq 2$, every invertible linear transformation of $M_{n}(K)$ is of the form (3.11) or is

$$
X \mapsto A X^{t} B \text { for some } A, B \in \mathrm{GL}_{n}(K) .
$$

Sketch of the proof. Here one takes $\widetilde{G}:=\mathrm{SL}_{m} \times \mathrm{SL}_{n}$ and $\widetilde{L}:=\mathrm{GL}_{m} \times \mathrm{GL}_{n}$ acting on the space $V$ of $m$-by- $n$ matrices via $\widetilde{\rho}(A, B) X=A X B^{t}$ and otherwise imitates the proofs of Corollaries 3.5 and 3.7 . 
Example 3.13 (Homogeneous polynomials of degree $d$ ). Assume char $K=0$ or $>d$ and continue the notation of Example 1.3. Viewing $K^{n}$ as the dual of a vector space, the representation $V$ becomes the vector space of homogeneous polynomials of degree $d$ in $n$ variables. As $\operatorname{Aut}(\Delta, \lambda)=\left\{\operatorname{Id}_{\Delta}\right\}$, Theorem 3.2 gives when $K$ is infinite: the collection of linear transformations of $V$ that preserve the set of $d$-th powers of nonzero linear forms is the compositum of $G$ and the scalar matrices in GL $(V)$. Compare [Sha83, Th. 10.5.5].

For $K$ of arbitrary characteristic, we can instead identify $V$ with the dual of $S^{d}\left(K^{n}\right)$ [Jan03, II.2.13-16]; we leave the explicit description in this case to the reader.

Example 3.14 (Exterior powers). Take $\widetilde{G}=\mathrm{SL}_{n}$ and $V=\bigwedge^{d} K^{n}$ for some $1 \leq d<$ $n$ as in Example 1.2, with $K$ infinite. Theorem 3.2 gives for $d \neq n / 2$ : the collection of linear transformations of $V$ that preserve the set of nonzero decomposable vectors is the compositum of $\mathrm{SL}_{n}$ and the scalar transformations. (In case $d=n / 2$, every linear transformation that preserves the decomposable vectors is as in the previous sentence or is the composition of such a transformation with a Hodge star operator.) Compare [Nem63, 3.1, 3.2], Wes64, or Gur97, Cor. 7.3].

The hypothesis on $V$ in Theorem 3.2 that $P$ is not exceptional-is weak enough that many other examples can also be treated readily. For example, one can recover the stabilizer of the decomposable tensors in $\bigotimes_{i=1}^{r} K^{n_{i}}$ as in [Wes67, Th. 3.8]. We also have the following:

Example 3.15 (Pure spinors). Take $\widetilde{G}=\operatorname{Spin}_{2 n}$ for some $n \geq 3$ (so $\widetilde{G}$ is of type $D_{n}$ ) and take $V$ to be a half-spin representation as defined in [Che97. or [Bou05, §VIII.13.4(IV)]. This representation is injective (i.e., $G=\widetilde{G}$ ) if $n$ is odd, and has kernel $\mu_{2}$ if $n$ is even; in this latter case, the image $G$ is called a half-spin or semi-spin group. The minimal elements in $V$ are the pure spinors as defined in [Che97, §3.1].

The representation $V$ is irreducible (regardless of the characteristic of $K$ ) because it is minuscule. As $\operatorname{Aut}(\Delta, \lambda)=\left\{\operatorname{Id}_{\Delta}\right\}$, Theorem 3.2 gives: if $K$ is infinite, the collection of linear transformations of $V$ that preserve the set of pure spinors is the compositum of $G$ and the scalar matrices.

Example 3.16 (Minimal nilpotents). Let $\widetilde{G}$ be a split, simple, and simply connected group and take $V=\operatorname{Lie}(\widetilde{G})$. This is a Weyl module for $\widetilde{G}$, and it is irreducible if char $K$ is very good for $\widetilde{G}$. The minimal elements in Lie $(\widetilde{G})$ are called minimal nilpotents.

As $\lambda$ is the highest root, $\operatorname{Aut}(\Delta, \lambda)=\operatorname{Aut}(\Delta)$. Theorem 3.2 gives that, for $K$ infinite, the collection of linear transformations of $\operatorname{Lie}(\widetilde{G})$ that preserve the minimal nilpotents is the compositum of the adjoint group $G$, the scalar transformations, and a copy of $\operatorname{Aut}(\Delta)$.

To summarize what we observed in this section: the minimal elements are stabilized by the normalizer of $G$ in GL $(V)$ (Example 3.1). We proved that in many cases the normalizer of $G$ is exactly the stabilizer of the minimal elements (Theorem 3.2), using Demazure's description of the automorphism group of projective homogeneous varieties. From this and $\$ 2$, one can read off the group of linear transformations that preserve the minimal elements in many cases. 


\section{The stabilizer in PGL $(V)$}

Theorem 3.2 has a clean reformulation in terms of subgroups of $\operatorname{PGL}(V)$. We maintain the hypotheses on $G$ and $V$ from $\$ 1$ Put $\bar{G}$ for the image $G / Z$ of $G$ in $\operatorname{PGL}(V)$.

Theorem 4.1. There are natural inclusions of smooth algebraic groups

$$
N_{\mathrm{PGL}(V)}(\bar{G}) \hookrightarrow \operatorname{Stab}_{\mathrm{PGL}(V)}(\mathscr{O}) \hookrightarrow \operatorname{Aut}(\mathscr{O}) .
$$

If $P$ is not exceptional, then both maps are isomorphisms. If $\bar{G}$ is simple and $V$ is irreducible, then the second map is an isomorphism.

Proof. We first claim that the group $N_{\mathrm{PGL}(V)}(\bar{G})$ is the semidirect product of $\bar{G}$ and the image of $\gamma(\operatorname{Aut}(\Delta, \lambda))$ in $\operatorname{PGL}(V)$. For this, the exact sequence $1 \rightarrow \mathbb{G}_{m} \rightarrow$ $\mathrm{GL}(V) \rightarrow \operatorname{PGL}(V) \rightarrow 1$ identifies $N_{\mathrm{PGL}(V)}(\bar{G})$ with the image of $N_{\mathrm{GL}(V)}\left(\mathbb{G}_{m} \cdot G\right)$ in $\operatorname{PGL}(V)$. As $\mathbb{G}_{m}$ and $G$ are the center and derived subgroup of $\mathbb{G}_{m} \cdot G$ respectively, $N_{\mathrm{GL}(V)}\left(\mathbb{G}_{m} \cdot G\right)=N_{\mathrm{GL}(V)}\left(\mathbb{G}_{m}\right) \cap N_{\mathrm{GL}(V)}(G)=N_{\mathrm{GL}(V)}(G)$. The claim follows from Proposition 2.2

The previous paragraph combines with the proof of Theorem 3.2 to give the existence of the arrows, as well as the statement that both are isomorphisms if $P$ is not exceptional. If $\bar{G}$ is simple and $V$ is irreducible (and $P$ is exceptional), then there is a larger simple group $\bar{G}^{\prime}$ contained in $\operatorname{PGL}(V)$ so that the stabilizer $P^{\prime}$ in $\bar{G}^{\prime}$ of a point in $\mathscr{O}$ is a parabolic subgroup that is not exceptional [Sei87, (8.1), $(8.14)]$, and we are done by the previous case.

\section{INTERLUdE: NONSPLIT GROUPS}

So far, we have assumed that the group $G$ is split. We now explain how to remove this hypothesis. Suppose for the duration of this section that $G$ is a semisimple group over $K$ with a faithful representation $\rho: G \rightarrow \operatorname{GL}(V)$ and that, after base change to a separable closure $K_{\text {sep }}$ of $K, \rho$ is irreducible or a Weyl module.

We can fix a pinning of $G \times K_{\text {sep }}$, a highest weight vector $v^{+} \in V \otimes K_{\mathrm{sep}}$, a parabolic $P:=\operatorname{Stab}_{G \times K_{\text {sep }}}\left(K_{\text {sep }} v^{+}\right)$, and a closed orbit $\mathscr{O} \in \mathbb{P}(V) \times K_{\text {sep }}$ as in $\$$.

Proposition 5.1. The closed $G$-orbit $\mathscr{O}$ is defined over $K$ and $\operatorname{Stab}_{\mathrm{GL}(V)}(\mathscr{O})=$ $N_{\mathrm{GL}(V)}(G)$.

Proof. For $\sigma \in \operatorname{Gal}\left(K_{\mathrm{sep}} / k\right)$, the action of $G$ on $V$ commutes with $\sigma$, so $\sigma(\mathscr{O})$ is a closed $G$-orbit in $\mathbb{P}(V)$ whose elements span $V \otimes K_{\text {sep }}$, ergo $\sigma\left(\mathscr{O}\left(K_{\text {sep }}\right)\right)=\mathscr{O}\left(K_{\text {sep }}\right)$. By the Galois criterion for rationality [Bor91, AG.14.4], $\mathscr{O}$ is defined over $K$. The group schemes $N_{\mathrm{GL}(V)}(G)$ and $\operatorname{Stab}_{\mathrm{GL}(V)}(\mathscr{O})$ are both defined over $K$, and the claimed equality is by Theorem 3.2 .

Suppose now that the representation $V \otimes K_{\text {sep }}$ is as in Table A We will prove in Propositions 8.1 9.1, and 11.1 that

$$
\operatorname{Stab}_{\mathrm{GL}(V)}(f) \subset \operatorname{Stab}_{\mathrm{GL}(V)}(\{f=0\})=N_{\mathrm{GL}(V)}(G)
$$

as group schemes over $K_{\text {sep }}$, and it follows from Proposition 5.1 that these relationships also hold over $K$. 


\section{REPRESENTATIONS WITH A 1-DIMENSIONAL RING OF INVARIANTS}

We have completed our study of linear transformations that preserve minimal elements, and we now move on to considering linear preserver problems (LPPs) as described in the introduction. We maintain the notation of $₫$, so $\widetilde{G}$ is a split semisimple algebraic group over the field $K$ and $\rho: \widetilde{G} \rightarrow \mathrm{GL}(V)$ is an irreducible representation or a Weyl module. The $d=2$ cases from Examples 1.2 and 1.3 are special in that the ring $K[V]^{G}$ of $G$-invariant polynomial functions on $V$ equals $K[f]$ for a nonconstant homogeneous polynomial $f$. (We say that $f$ is $G$-invariant if every $g \in G\left(K_{\mathrm{alg}}\right)$ preserves $f$, where we use the typical algebraist's definition that an element $g \in G(K)$ preserves $f$ if $f \circ g=f$ as polynomials.)

The basic facts about this situation are given by the following proposition, which is well known for $K=\mathbb{C}$; see e.g. Pop80, Prop. 12]. The quotient $V / G$ is defined to be the variety $\operatorname{Spec} K[V]^{G}$.

Proposition 6.1. The following are equivalent:

(1) $\operatorname{dim} V / G=1$.

(2) There is a dense open $G\left(K_{\text {alg }}\right)$-orbit in $\mathbb{P}(V)\left(K_{\text {alg }}\right)$ but not in $V \otimes K_{\text {alg }}$.

(3) $K[V]^{G}=K[f]$ for some homogeneous $f \in K[V] \backslash K$.

(4) $V / G$ is isomorphic to the affine line $\mathbb{A}^{1}$.

Proof. Assuming (1), Theorem 4 in $\left[\right.$ Sch00 gives that the fraction field of $K[V]^{G}$ is $K(f)$ for some homogeneous $f$, and as in the proof of that theorem we deduce (3). (3) implies (4) because the polynomial $f$ (as an element of $K[V]$ ) is transcendental over $K$, and (4) trivially implies (1).

Now suppose (2). Recall that there is a $G$-invariant dense subset $U$ of $V \otimes K_{\text {alg }}$ such that two elements of $U$ have the same image in $V / G$ iff they are in the same $G\left(K_{\text {alg }}\right)$-orbit. So, if $L$ is a line in $V$ that is in the open orbit in $\mathbb{P}(V)\left(K_{\text {alg }}\right)$, then $G\left(K_{\text {alg }}\right) \cdot L$ contains a nonempty open subset of $V$, hence contains a nonempty open subset of $U$. It follows that the map $L \rightarrow V / G$ is dominant, hence that $\operatorname{dim} V / G$ is 0 or 1 . But for an orbit $X$ of maximal dimension in $V$, we have $\operatorname{dim} V / G=\operatorname{dim} V-\operatorname{dim} X$, so if $\operatorname{dim} V / G$ is 0 , there is a dense orbit in $V \otimes K_{\text {alg }}$. (1) is proved.

Finally suppose (3) holds; we prove (2). The map $f: V \otimes K_{\mathrm{alg}} \rightarrow K_{\mathrm{alg}}$ is $G$-invariant and nonconstant, so there is no dense orbit in $V \otimes K_{\text {alg. As }} f$ is homogeneous and separates the $G\left(K_{\text {alg }}\right)$-orbits in $U$, it follows that the dense image of $f^{-1}\left(K_{\mathrm{alg}}^{\times}\right) \cap U$ in $\mathbb{P}(V)$ is a single (open) $G\left(K_{\mathrm{alg}}\right)$-orbit.

Representations where the conditions in the proposition hold are closely related to the prehomogeneous vector spaces studied in [SK77], the $\theta$-groups studied by Vinberg as in [PV94, and the internal Chevalley modules from ABS90.

All pairs $(G, V)$ with a 1-dimensional ring of invariants, $G$ simple, and $K=\mathbb{C}$ are listed on pages 260-262 of [PV94]. In $\S ₫ 8$ and 9] we will solve the LPP for the representations listed in Table $\mathrm{A}$. That table does not include all possibilities from [PV94, and we make some remarks about the remaining entries in $\$ 10$

Internal Chevalley modules over $\mathbb{Z}$. Let $H$ be a split simple linear algebraic group over $\mathbb{Z}$ and fix a fundamental dominant weight $\omega$ of $H$ with respect to some maximal torus $T$. The choice of $\omega$ defines a parabolic subgroup $P$ of $H$ and a Levi subgroup of $P$ (generated by $T$ and the root subgroups for roots orthogonal to $\omega$ ). We define $\widetilde{G}$ to be the derived subgroup of $L$ and $V$ to be the submodule 
of $\operatorname{Lie}(H)$ generated by the root subalgebras for roots $\alpha$ with $\langle\alpha, \omega\rangle=1$; it is a free $\mathbb{Z}$-module of finite rank. Following the notation of $₫ \mathbb{1}$, we define $G$ to be the (scheme-theoretic) image of $\widetilde{G}$ in $\operatorname{GL}(V)$.

By Ses77, Th. 2], the ring $\mathbb{Z}[V]^{G}$ of $G$-invariant polynomial functions in $\mathbb{Z}[V]$ is finitely generated. We simply write $K[V]^{G}$ for the ring of $(G \times K)$-invariant polynomial functions on $V \otimes K$. There is a natural inclusion $\mathbb{Z}[V]^{G} \otimes K \rightarrow K[V]^{G}$, but it need not be surjective when $K$ has prime characteristic; see Example 6.3 .

Proposition 6.2. Let $G$ and $V$ be as in the previous two paragraphs and suppose that $\operatorname{dim} \mathbb{C}[V]^{G}=1$. Then:

(1) For every field $K, \operatorname{dim} K[V]^{G}=1$.

(2) $\mathbb{Z}[V]^{G}=\mathbb{Z}[f]$ for a nonzero, indivisible polynomial $f$ that is determined up to sign.

Proof. The map $\mathbb{Z}[V]^{G} \otimes \mathbb{Q} \rightarrow \mathbb{Q}[V]^{G}$ is an isomorphism because $\mathbb{Q}$ is flat over $\mathbb{Z}$ Ses77, Lemma 2], and similarly for $\mathbb{Q}[V]^{G} \otimes_{\mathbb{Q}} \mathbb{C} \stackrel{\sim}{\longrightarrow} \mathbb{C}[V]^{G}$. Hence the fiber of $\operatorname{Spec}\left(\mathbb{Z}[V]^{G}\right) \rightarrow \operatorname{Spec}(\mathbb{Z})$ over the generic point has dimension 1 and upper semicontinuity gives $\operatorname{dim} K[V]^{G} \geq 1$ for all $K$. On the other hand, Th. 2f and the remark on p. 560 of [ABS90] give that $\operatorname{dim} K[V]^{G} \leq 1$, proving (11).

Proposition 6.1 gives that $\operatorname{Spec}\left(K[V]^{G}\right)$ is isomorphic to $\mathbb{A}^{1}$ for every $K$; hence $\mathbb{Z}[V]^{G}$ is "locally polynomial" by KM78, i.e., for every prime $p, \mathbb{Z}_{(p)}[V]^{G}$ is isomorphic to polynomials in one variable over $\mathbb{Z}_{(p)}$. Claim (2) now follows by ES72, 3.12].

In order to determine a formula for (or the degree of) the generator $f$ of $\mathbb{Z}[V]^{G}$, it suffices to do so for its image in $\mathbb{C}[V]^{G}$. The degree can be looked up in PV94 or [Kac80, Table II] or can be calculated using [SK77, p. 65, Prop. 15].

The representations in Table $\mathbf{A}$. We now verify that the representations in Table $\mathrm{A}$ are irreducible. If $K=\mathbb{C}$, then this is well known, so we assume that char $K$ is a prime and we apply results from ABS90. Fix a particular $G, V$, and $K$ from Table $\mathrm{A}$ for consideration; we can obtain $G$ as in the previous subsection by taking $H$ to be simply connected of type $C_{n} ; D_{n} ; E_{7} ; A_{2 n-1} ; B$ or $D ; G_{2} ; E_{6}$; $F_{4} ; E_{7} ; E_{8}$; and $B$ or $D$ respectively. By ABS90. (using our assumption on char $K$ in case $H$ has roots of different lengths), $V$ is an irreducible representation of $G$, as claimed.

Furthermore, in each of these cases, $\operatorname{dim} \mathbb{C}[V]^{G}=1$ by SK77 or Kac80, $\left.\S 2\right]$, so by Proposition 6.2(1) $\operatorname{dim} K[V]^{G}=1$. We claim that the image of the polynomial $f$ from Proposition 6.2 (2) generates $K[V]^{G}$ as a $K$-algebra. To see this, note that $K[V]^{G}=K[h]$ for some nonzero homogeneous $h$, so $f=c h^{r}$ for some $c \in K^{\times}$and $r \geq 1$. Now it suffices to verify that $f$ is irreducible in $K[V]$ (as is well known for the determinant from line 4) or to find an element $v \in V \otimes K_{\mathrm{alg}}[t]$ such that $f(v) \in K_{\text {alg }}^{\times} t$ (as can be done from (8.3) for lines 1-5 or from the formula for $f$ from [Bro69, p. 87] or [Fer72, p. 314] for lines 6-11); the claim is proved.

Example 6.3 (Binary cubics). Suppose char $K \neq 2,3$ and consider the vector space $V$ of binary cubic forms; it is the irreducible representation $V=S^{3}\left(\left(K^{2}\right)^{*}\right)$ of $G=\mathrm{SL}_{2}$ from line 5 of Table $\mathrm{A}$. In the notation of the three preceding paragraphs, one takes $H$ split of type $G_{2}$. The maps that send $(x, y)$ to $x^{3}, x^{2} y, x y^{2}, y^{3}$ are a 
basis for the $R$-module $V$, and a formula for $f$ is given in [Web95, §46, (10)] or Gur64, (14.33)]:

$$
f\left(a_{0} x^{3}+a_{1} x^{2} y+a_{2} x y^{2}+a_{3} y^{3}\right)=a_{1}^{2} a_{2}^{2}+18 a_{0} a_{1} a_{2} a_{3}-4 a_{0} a_{2}^{3}-4 a_{1}^{3} a_{3}-27 a_{0}^{2} a_{3}^{2}
$$

This is the discriminant of the cubic form.

We remark that binary cubic forms (and $f$ ) can be identified with cubic algebras (and their discriminant algebras) as described in [GGS02, §4] or HM00]. Furthermore, this representation is irreducible also in case char $K=2$ by Steinberg's tensor product theorem [Jan03, II.3.17]. We ignore these variations below.

The formula for $f$ in (6.4) illustrates how the map $\mathbb{Z}[V]^{G} \otimes K \rightarrow K[V]^{G}$ need not be surjective: when $K$ has characteristic 2 , the image of $f$ is $\left(a_{1} a_{2}+a_{0} a_{3}\right)^{2}$. A similar phenomenon happens for all the representations on lines $6-11$ of Table $\mathrm{A}$, as can be seen from the general formula for $f$ in [Bro69] or [Fer72].

Example 6.5. The group $\widetilde{G}=\mathrm{SL}_{2} \times \mathrm{SO}_{n}$ for $n \geq 4$ acts naturally on $V=K^{2} \otimes K^{n}$. Suppose char $K \neq 2$. Then $V$ is irreducible, and in the notation of this section, one takes $H=\operatorname{Spin}_{n+4}$. We may identify $V$ with the 2 -by- $n$ matrices so that $\widetilde{G}$ acts via $\rho\left(g_{1}, g_{2}\right) X=g_{1} X g_{2}^{t}$. There is a symmetric $S \in \mathrm{GL}_{n}(K)$ so that the $K$-points of $\mathrm{SO}_{n}$ are the $g_{2} \in \mathrm{SL}_{n}(K)$ such that $g_{2}^{t} S g_{2}=S$. It follows that the polynomial map $f: V \rightarrow K$ defined by $f(X):=\operatorname{det}\left(X S X^{t}\right)$ is invariant under $\widetilde{G}$. It generates $\mathbb{C}[V]^{G}$ as argued in $\left[\right.$ SK77, pp. 109, 110], so $K[V]^{G}=K[f]$.

In the smallest case $n=4$, one can equivalently take $\widetilde{G}=\mathrm{SL}_{2} \times \mathrm{SL}_{2} \times \mathrm{SL}_{2}$ and $V=K^{2} \otimes K^{2} \otimes K^{2}$. In that case, $f$ is Cayley's hyperdeterminant defined in Cay45. It appears, for example, in quantum information theory to measure the entanglement of a 3-qubit system [MW02].

TABLE A. Some representations $V$ of groups $\widetilde{G}$ so that $f$ generates the ring of all polynomials on $V$ that are invariant under $\widetilde{G}$. In these cases, we calculate the subgroup $\operatorname{Stab}_{\mathrm{GL}(V)}(f)$ of $\mathrm{GL}(V)$.

\begin{tabular}{|c|c|c|c|c|c|c|}
\hline \# & $\widetilde{G}$ & $V$ & $\operatorname{dim} V$ & $f$ & $\operatorname{deg} f$ & $\operatorname{char} K$ \\
\hline 1 & $\mathrm{SL}_{n}$ & $S^{2}\left(K^{n}\right)$ & $\left(\begin{array}{l}n \\
2\end{array}\right)+n$ & $\operatorname{det}$ & $n$ & $\neq 2$ \\
\hline 2 & $\begin{array}{c}\mathrm{SL}_{n} \\
(n \text { even, } n>4)\end{array}$ & $\wedge^{2}\left(K^{n}\right)$ & $\left(\begin{array}{l}n \\
2\end{array}\right)$ & $\mathrm{Pf}$ & $n / 2$ & \\
\hline 3 & $\mathrm{E}_{6}^{\mathrm{sc}}$ & minuscule & 27 & see Jac68 p. 358] & 3 & \\
\hline 4 & $\mathrm{SL}_{n} \times \mathrm{SL}_{n}$ & $M_{n}$ & $n^{2}$ & $\operatorname{det}$ & $n$ & \\
\hline 5 & $\mathrm{SO}_{n}(n \geq 3)$ & $K^{n}$ & $n$ & & 2 & $\left\{\begin{array}{l}\neq 2 \text { if } n \\
\text { odd }\end{array}\right.$ \\
\hline 6 & $\mathrm{SL}_{2}$ & $\begin{array}{l}\text { binary } \\
\text { cubics }\end{array}$ & 4 & see Example 6.3 & 4 & $\neq 2,3$ \\
\hline 7 & $\mathrm{SL}_{6}$ & $\wedge^{3}\left(K^{6}\right)$ & 20 & see 9.4 & : & : \\
\hline 8 & $\mathrm{Sp}_{6}$ & $\wedge_{0}^{3}\left(K^{6}\right)$ & 14 & see 9.4 & : & : \\
\hline 9 & $\operatorname{Spin}_{12}$ & half-spin & 32 & see Igu70 p. 1012] & : & : \\
\hline 10 & $\mathrm{E}_{7}^{\mathrm{Sc}}$ & minuscule & 56 & see Fer72, Bro69 & : & : \\
\hline 11 & $\begin{array}{c}\mathrm{SL}_{2} \times \mathrm{SO}_{n} \\
(n \geq 4)\end{array}$ & $K^{2} \otimes K^{n}$ & $2 n$ & see Example 6.5 & 4 & $\neq 2,3$ \\
\hline
\end{tabular}




\section{Transformations that PRESERVe minimal Elements and $f$}

We maintain the assumptions of the previous sections, and from here on we assume furthermore that $V$ is irreducible and the ring $K[V]^{G}$ of $G$-invariant polynomial functions on $V$ is generated by a nonconstant homogeneous element that we denote by $f$. Since $V$ is an irreducible representation of $G$ and $f$ is not constant, the subspace consisting of $r \in V$ such that $f(r+v)=f(v)$ for all $v \in V$ must be zero. It follows easily from this that every linear transformation preserving $f$ is invertible, as noted in [Wat83, §1]. Hence the collection of linear transformations $\phi$ of $V$ that preserve $f$ is the group of $K$-points of the closed sub-group-scheme $\operatorname{Stab}_{\mathrm{GL}(V)}(f)$ of $\mathrm{GL}(V)$. We call it the preserver of $f$; the classical linear preserver problem is to determine the $K$-points of this group.

Lemma 7.1. If $K$ is algebraically closed, then

$$
N_{\mathrm{GL}(V)}(G)^{\circ}(K) \cap \operatorname{Stab}_{\mathrm{GL}(V)}(f)(K)=G(K) \cdot \mu_{\mathrm{deg} f}(K) .
$$

Proof. By Corollary 2.4, every element of $N_{\mathrm{GL}(V)}(G)^{\circ}(K)$ is a product $g z$ for some $g \in G(K)$ and $z \in K^{\times}$, and the claim is clear.

In the notation of (2.5), the equation

$$
f(\widetilde{\rho}(g) v)=\chi(g) f(v) \quad \text { for all } v \in V \otimes K_{\text {alg }}
$$

defines a homomorphism $\chi: \widetilde{L} \rightarrow \mathbb{G}_{m}$. Corollary 2.6 immediately gives:

Lemma 7.2. Assuming (2.5), the elements of $N_{\mathrm{GL}(V)}(G)^{\circ}(K)$ that preserve $f$ are $\widetilde{\rho}(g)$ for $g \in \widetilde{L}(K)$ such that $\chi(g)=1$.

As to the nonidentity component of $N_{\mathrm{GL}(V)}(G)$, we have:

Lemma 7.3. There is a homomorphism $\phi: \operatorname{Aut}(\Delta, \lambda) \rightarrow \mathbb{G}_{m}$ such that $f(\gamma(\pi) v)=$ $\phi(\pi) f(v)$ for all $\pi \in \operatorname{Aut}(\Delta, \lambda)$ and $v \in V \otimes E$ for every extension $E$ of $K$.

Proof. For a fixed $\pi \in \operatorname{Aut}(\Delta, \lambda)$, define $f_{\pi} \in K[V]$ via $f_{\pi}(v):=f(\gamma(\pi) v)$. As

$$
f_{\pi}(g v)=f(\gamma(\pi) g v)=f((i(\pi)(g)) \gamma(\pi) v)=f_{\pi}(v) \text { for all } v \in V \otimes E,
$$

and $f_{\pi}$ and $f$ are homogeneous of the same degree in $K[V]^{G}$, we deduce that $f_{\pi}=\phi(\pi) f$ for some scalar $\phi(\pi) \in K$. As $f$ is nonzero on $V, f_{\pi}$ is also nonzero, hence $\phi(\pi) \in K^{\times}$.

\section{Lines $1-5$ of TABle $\mathrm{A}$}

For each of the polynomials $f$ appearing in lines $1-5$ of Table $\mathrm{A}$ we will determine the linear transformations of $V$ that preserve $f$. We prove the following, which is a formal version of an imprecise observation made in [Mar62, p. 840].

Proposition 8.1. For the representations in lines 1-5 of Table A, every linear transformation of $V$ that preserves $f$ belongs to $N_{\mathrm{GL}(V)}(G)(K)$.

Because we know so much about these representations, we can check this by hand in each case. This is well known for line 4, is done for line 1 in Eat69, and a similar argument using the generic minimal polynomial defined in Jac68, Ch. VI] or Gar04 works for lines 2 and 3. Alternatively, the proposition follows easily from the following: 
Lemma 8.2. Suppose $K$ is infinite. For the representations in lines 1-5 of Table A, nonzero $v \in V$, and an indeterminate $t$, we have: $v$ is minimal if and only if $\operatorname{deg} f\left(t v+v^{\prime}\right) \leq 1$ for all $v^{\prime} \in V$.

Again, this claim can be checked by hand in each case. This is trivial for line 5 and is done for line 4 in [MP59, Lemma 3.2] — note that the difficult direction in that paper is "if". We give a more uniform proof of the "if" direction based on RRS92].

Proof of Lemma 8.2, "if". Whether or not $v$ is minimal is unchanged upon enlarging $K$, and the same is true for the other condition (because $K$ is infinite), so we may assume that $K$ is algebraically closed. The representation $G \rightarrow \operatorname{GL}(V)$ is not only a representation as in $\$ 6$, it is furthermore of the type considered in [RRS92 or Rub92, §5]. In particular, there is a sequence $u_{1}, \ldots, u_{d}$ of weight vectors in $V$ so that every element of $V$ is in the $G(K)$-orbit of some $\sum_{i=1}^{r} c_{i} u_{i}$ for $c_{i} \in K^{\times}$[RRS92, Th. 1.2(a)]; an element is minimal if and only if it is in the orbit of $c_{1} u_{1}$ for some $c_{1} \in K^{\times}$; and $f$ vanishes on $\sum_{i=1}^{r} c_{i} u_{i}$ if and only if $r<d$ (ibid., Prop. 2.15(b)).

The number $d$ is calculated from root system data (ibid., p. 658), but in each case we see that it equals the degree of the invariant polynomial $f$ computed as described in $\$ 6$. We claim that the restriction of $f$ to the span of the $u_{i}$ is given by

$$
f\left(\sum_{i=1}^{d} c_{i} u_{i}\right)=c \prod_{i=1}^{d} c_{i} \quad \text { for some } c \in K^{\times} .
$$

Indeed, the normalizer of $T$ in $G$ permutes the $u_{i}$ arbitrarily (ibid., Th. 2.1), so the monomials appearing with a nonzero coefficient in the formula for the restriction of $f$ are stable under the obvious action by the symmetric group on $d$ letters. The condition that $f\left(\sum_{i=1}^{r} c_{i} u_{i}\right)$ with $c_{i} \in K^{\times}$vanishes if and only if $r<d$ together with the degree of $f$ being $d$ implies the claimed formula.

Finally, if $v$ is nonminimal, then it is in the orbit of $\sum_{i=1}^{r} c_{i} u_{i}$ for some $r>1$, and it is easy to produce a $v^{\prime}$ so that $\operatorname{deg} f\left(t v+v^{\prime}\right)>1$; this settles the "if" direction.

Proof of Proposition 8.1. Any linear transformation $\phi$ that preserves $f$ by definition also preserves $f$ over every extension of $K$. Hence, by Lemma 8.2, $\phi$ preserves minimal elements in $V \otimes K_{\text {alg }}$, i.e., $\phi$ belongs to $\operatorname{Stab}_{\mathrm{GL}(V)}(\mathscr{O})$, which equals $N_{\mathrm{GL}(V)}(G)$ by Theorem 3.2 .

Remark 8.4. Lines 1, 2, and 4 of Table $\mathrm{A}$ have in common that $V$ can be endowed with a bilinear multiplication that is "strictly power associative", and so $V$ has a generic characteristic polynomial as mentioned above. Write $E_{r}$ for the coefficient of the characteristic polynomial that is a homogeneous function on $V$ of degree $r$, so that $E_{d}=f$. A uniform argument as in Lemma 8.2 shows that the preserver in $\mathrm{GL}(V)$ of $E_{r}$ for each $r \in\{3, \ldots, d-1\}$ is contained in $\operatorname{Stab}_{\mathrm{GL}(V)}(\mathscr{O})$. We omit the details, but the interested reader can find a precise description of the preserver of $E_{r}$ in [Gur97, Cor. 6.5] for symmetric matrices (line 1), [MW60] or [Gur97, Cor. 7.7] for alternating matrices (line 2), and [MP59], Bea70, or [Wat95, Cor. 1] for square matrices (line 4). (For line 3, one also has a generic characteristic polynomial, but $f$ is the only coefficient of degree $\geq 3$.) 
Remark 8.5. Lines $1-5$ of the table do not exhaust all the representations considered by RRS92. The ones we have omitted lack a $G$-orbit of codimension 1 (ibid., Prop. 3.12), yet there is an open $G$-orbit in $\mathbb{P}(V)$. Hence every $G$-invariant polynomial on $V$ is constant.

We can now determine the subgroup of $\operatorname{GL}(V)$ of elements that preserve $f$. Our first result concerns symmetric matrices as in line 1 of the table. Compare [Fro97, §7.III], [Eat69, Th. 1], Lim79, Wat87, Th. 6.7], or [Gur97, Cor. 6.3].

Corollary 8.6 (Symmetric matrices). For $n \geq 2$ and $K$ of characteristic $\neq 2$, every linear transformation $\phi$ of $\operatorname{Symm}_{n}(K)$ that preserves the determinant is of the form (3.6) where $r^{n} \operatorname{det}(P)^{2}=1$.

Proof. Combine Proposition 8.1, Lemma 7.2, and Corollary 3.5.

The next results concern alternating matrices as in line 2 of the table. Compare [MW60, Th. 3], Wat87, Th. 5.7], or [Gur97, Cor. 7.4].

Corollary 8.7 (Alternating $n$-by-n matrices). For even $n \geq 6$, every linear transformation $\phi$ of $\operatorname{Skew}_{n}(K)$ that preserves the Pfaffian is of the form (3.6) where $r^{n / 2} \operatorname{det}(P)=1$.

Corollary 8.8 (Alternating 4-by-4 matrices). Every linear transformation of Skew $_{4}(K)$ that preserves the Pfaffian is of the form (3.6) or (3.8) where $r^{n / 2} \operatorname{det}(P)$ $=1$

Proof. In view of Lemma 7.3, it suffices to pick some $X \in \operatorname{Skew}_{4}(K)$ with $\operatorname{Pf}(X) \neq 0$ and verify that $\operatorname{Pf}\left(X^{*}\right)=\operatorname{Pf}(X)$ for $*$ as in (3.9).

We now determine the linear transformations that preserve the determinant. This is the case famously treated by Frobenius in [Fro97, §7.I] and Dieudonné in [Die49, and also in [MM59a, Th. 2] and Wat87, Th. 4.2].

Corollary 8.9 (Square matrices). Every linear transformation of $M_{n}(K)$ that preserves the determinant is of the form (3.11) or (3.12) where $\operatorname{det}(A B)=1$.

Every minuscule representation $V$ of a group $G$ of type $E_{6}$ has a nonzero $G$ invariant cubic form $f$, and $G$-invariance uniquely determines $f$ up to multiplication by an element of $K^{\times}$. For the following result, compare [SV00, 7.3.2] (for char $K \neq$ 2,3 ) or [Asc87a, 5.4]. The analogous (and a priori coarser) result for Lie algebras is Lur01, 5.5.1].

Corollary $\mathbf{8 . 1 0}$ (Minuscule representation of $E_{6}$ ). In the notation of the preceding paragraph, the preserver of $f$ in $\mathrm{GL}(V)$ is $G(K)$.

Proof. Since $\operatorname{Aut}(\Delta, \lambda)=1$ and $\mu_{3}$ is in the center of $G$, Lemma 7.1 gives the claim.

Commuting with the adjoint. For the representations considered in this section, one has a notion of a "classical adjoint" adj: $V \rightarrow V$, which is a polynomial map of degree $(\operatorname{deg} f)-1$. For lines 1,2 , and 4 of the table, the papers [Sin83] and [CLT87] compute the linear transformations on $V$ that commute with this map. We can do the same for line 3 , where $G$ is the simply connected split group of type $E_{6}$. The $\operatorname{group} \operatorname{Aut}(\Delta)$ is $\mathbb{Z} / 2 \mathbb{Z}$ and we write $\pi$ for the nonzero element; the subgroup of $G$ of elements fixed by $i(\pi)$ is a split group of type $F_{4}$ [CG06, 7.3], which we denote simply by $F_{4}$. The center of $G$ is a copy of $\mu_{3}$. We find: 
Corollary 8.11. If char $K \neq 2,3$, then the subgroup of $\mathrm{GL}(V)$ of elements commuting with the adjoint is $F_{4}(K) \cdot \mu_{3}(K)$.

Proof. The minimal elements are precisely the nonzero $v \in V$ so that adj $v=0$ [CG06. 7.10], so any element of $\mathrm{GL}(V)$ that preserves adj necessarily preserves minimal elements and hence belongs to $\mathbb{G}_{m} . G$. (One could alternatively deduce this using the identity $\operatorname{adj}(\operatorname{adj} v)=f(v) v$.) Further, for any $g \in G$ and $c \in \mathbb{G}_{m}$, we have $\operatorname{adj}(c g v)=c^{2} i(\pi)(g) \operatorname{adj}(v)$ CG06, 7.9]; hence such a $c g$ commutes with adj if and only if $i(\pi)(g)=c^{-1} g$. In particular, $c$ belongs to $G$ and so is a cube root of unity. That is, the subgroup $H$ of $\mathrm{GL}(V)$ of elements commuting with the adjoint is contained in $G$, and the image of $H$ in the adjoint group $G / \mu_{3}$ is contained in the subgroup fixed by $i(\pi)$.

As $F_{4} \times \mu_{3}$ is obviously contained in $H$, it suffices to show that its image in $G / \mu_{3}$ is the subgroup fixed by $i(\pi)$. But this subgroup is connected reductive with Lie algebra of type $F_{4}$ [CG06, 7.3], hence is the same as the image of $F_{4}$. This proves the claim.

\section{Lines $6-11$ of TABle A}

The representations on lines $6-11$ of Table $\mathrm{A}$ are all of the form considered in [Röh93], [Fer72, and Mey68. In particular, the ring of $G$-invariant polynomials on $V$ is generated by a homogeneous polynomial $f$ of degree 4 . These representations appear, for example, when studying electromagnetic black hole charges in various supergravity theories; see $\mathrm{BDF}^{+} 12$. We suppose in this section that the characteristic of $K$ is $\neq 2,3$. The assumption that the characteristic is $\neq 2$ is so that we may apply the results of Röh93, and the assumption that the characteristic is $\neq 3$ is a convenience so that we may apply the results of [Hel12]. We will prove:

Proposition 9.1. For the representations in lines 6-11 of Table $\mathrm{A}$, every element of $\mathrm{GL}(V)$ that preserves $f$ belongs to $N_{\mathrm{GL}(V)}(G)(K)$.

In view of our assumption on the characteristic, we are free to abuse notation and multilinearize $f$ to obtain a symmetric 4-linear form that we also denote by $f$. Further, for each of these representations, there is a nondegenerate skew-symmetric bilinear form $\langle$,$\rangle on V$ that is invariant under $G$. This allows us to define a trilinear map $t: V \times V \times V \rightarrow V$ implicitly by the equation

$$
\left\langle t\left(x_{1}, x_{2}, x_{3}\right), x_{4}\right\rangle=f\left(x_{1}, x_{2}, x_{3}, x_{4}\right) \quad \text { for } x_{1}, x_{2}, x_{3}, x_{4} \in V .
$$

For each $x \in V$, we define a symmetric bilinear form $b_{x}$ on $V$ via $b_{x}\left(v_{1}, v_{2}\right)=$ $f\left(x, x, v_{1}, v_{2}\right)$. We have:

Lemma 9.2. $x$ is a minimal element if and only if the dimension of the radical of $b_{x}$ is $(\operatorname{dim} V)-1$.

Proof. Line 6 is the representation from Example 6.3, for which we may check the claim of the lemma by hand. So assume $G$ and $V$ come from one of the lines 7-11; we may apply results from $\S 3-4$ of [Hel12].

The radical of $b_{x}$ has codimension 1 if and only if it is the subspace $y^{\perp}$ of $V$ of vectors orthogonal (relative to $\langle$,$\rangle ) to some nonzero y \in V$. That is, if and only if there is a nonzero $y \in V$ such that $f\left(x, x, y^{\perp}, z\right)=0$ for all $z \in V$. (For "only if", one needs to know that $b_{x}$ is nonzero for $x$ nonzero, which is Lemma 14 in ibid.) In turn, this is equivalent to: there is a nonzero $y \in V$ such that $t(x, x, z) \in K y$ for 
every $z \in V$. But by ibid., Propositions 18 and 20, that is the same as asking for $x$ to be minimal.

Proof of Proposition 9.1. Suppose $\phi$ preserves $f$. It defines an isometry between the bilinear forms $b_{x}$ and $b_{\phi(x)}$ for all $x \in V$. Now apply Lemma 9.2 to deduce that $\phi$ belongs to $\operatorname{Stab}_{\mathrm{GL}(V)}(\mathscr{O})$, and hence to $N_{\mathrm{GL}(V)}(G)$ by Theorem 3.2 .

We now determine the preserver of the discriminant of binary cubic forms as in line 6 or Example 6.3. We omit the details in the proofs of this corollary and the following items because they are entirely similar to earlier proofs.

Corollary 9.3 (Binary cubics). Every linear transformation on the vector space of cubic forms $K^{2} \rightarrow K$ that preserves the discriminant is of the form $q \mapsto c q \circ g$ for some $c \in K^{\times}$and $g \in \mathrm{GL}_{2}(K)$ such that $c^{4}(\operatorname{det} g)^{6}=1$.

Line 7 of the table concerns an $\mathrm{SL}_{6}$-invariant quartic form $f$ on $\bigwedge^{3} K^{6}$, for which a (complicated-looking) formula is given in [SK77, p. 83]; we now give an alternative presentation. Write $K^{6}$ as a direct sum $V_{2} \oplus V_{4}$, where $V_{d}$ has dimension $d$. There is a natural inclusion $w: V_{2} \otimes\left(\bigwedge^{2} V_{4}\right) \rightarrow \bigwedge^{3} K^{6}$ given by $(c, x) \mapsto c \wedge x$. Amongst the line of invariant quartic forms on $\wedge^{3} K^{6}$, there is an element $f$ so that, with respect to a fixed basis $a, b$ of $V_{2}$, we have

$$
f(w(a \otimes x+b \otimes y))=\langle x, y\rangle^{2}-4 \operatorname{Pf}(x) \operatorname{Pf}(y),
$$

where $\langle x, y\rangle$ denotes the coefficient of $t$ in $\operatorname{Pf}(x+t y)$, i.e., the polarization of the $\mathrm{SL}\left(V_{4}\right)$-invariant quadratic form Pf. (To check the claim of (9.4), one can either use the formula for $f$ in [SK77] or one can observe that $f w$ is a nonzero quartic form on $V_{2} \otimes\left(\bigwedge^{2} V_{4}\right)$ that is invariant under $\mathrm{SL}\left(V_{2}\right) \times \mathrm{SL}\left(V_{4}\right)$, that there is a unique line of such forms if $K=\mathbb{C}$, and that the right side of (9.4) gives such a form.) As every $\mathrm{SL}_{6}(K)$-orbit in $\bigwedge^{3} K^{6}$ meets the image of $w$ by [Rev79, Lemma 2.2], equation (9.4) is enough to specify $f$ on $\bigwedge^{3} K^{6}$.

Corollary 9.5. Every linear transformation of $\bigwedge^{3}\left(K^{6}\right)$ that preserves the invariant quartic form is of the form

(9.6) $v_{1} \wedge v_{2} \wedge v_{3} \mapsto c\left(g v_{1} \wedge g v_{2} \wedge g v_{3}\right)$ for $c \in K^{\times}, g \in \mathrm{GL}_{6}$ with $c^{4}(\operatorname{det} g)^{2}=1$

or the composition of a Hodge star operator with a transformation as in (9.6).

Regarding line 8 of the table, recall that $\mathrm{Sp}_{6}$ is defined as the subgroup of $\mathrm{GL}_{6}$ leaving a particular nondegenerate skew-symmetric bilinear form $b$ invariant on its (tautological) representation $K^{6}$. We write $\bigwedge_{0}^{3}\left(K^{6}\right)$ for the kernel of the contraction map $\bigwedge^{3}\left(K^{6}\right) \rightarrow K^{6}$; cf. [FH91, §17.1]. The restriction of the $\mathrm{SL}_{6}$-invariant quartic form on $\Lambda^{3} K^{6}$ to $\bigwedge_{0}^{3}\left(K^{6}\right)$ gives an $\mathrm{Sp}_{6}$-invariant quartic form.

We define $\mathrm{GSp}_{6}$ to be the subgroup of $\mathrm{GL}_{6}$ of transformations that scale the bilinear form $b$ by a factor in $K^{\times}$; it is isomorphic to $\left(\operatorname{Sp}_{6} \times \mathbb{G}_{m}\right) / \mu_{2}$.

Corollary 9.7. Every linear transformation of the space $\bigwedge_{0}^{3}\left(K^{6}\right)$ that preserves the invariant quartic is of the form

$$
v_{1} \wedge v_{2} \wedge v_{3} \mapsto c\left(g v_{1} \wedge g v_{2} \wedge g v_{3}\right) \quad \text { for some } c \in K^{\times} \text {and } g \in \operatorname{GSp}_{6}(K)
$$

with $c^{4}(\operatorname{det} g)^{2}=1$. 
For the representations on lines 9 and 10, we will prove a result under the assumption that $K$ contains a square root of -1 . Alternatively, we could eliminate this hypothesis at the cost of defining a reductive envelope $\widetilde{L}$ of $G$ as we defined $\mathrm{GSp}_{6}$ for $\mathrm{Sp}_{6}$ above, i.e., as in (2.5).

We write $\mathrm{HSpin}_{12}$ for the image $G$ of $\operatorname{Spin}_{12}$ under a half-spin representation.

Corollary 9.8. Suppose $K$ contains a square root of -1 . Then the subgroup of $\mathrm{GL}_{32}(K)$ of transformations that preserve the $\mathrm{HSpin}_{12}$-invariant quartic form is $\operatorname{HSpin}_{12}(K) \cdot \mu_{4}(K)$.

For the next result, compare [Spr06, Cor. 2.6(i)] or [Hel12, §10]. Those proofs are based on versions of Corollary 8.10 for $\mathrm{E}_{6}$, but our proof does not refer to $\mathrm{E}_{6}$.

Corollary 9.9. Suppose $K$ contains a square root of -1 . Then the subgroup of $\mathrm{GL}_{56}(K)$ of transformations that preserve the $\mathrm{E}_{7}^{\mathrm{sc}}$-invariant quartic form is $\mathrm{E}_{7}^{\mathrm{sc}}(K)$. $\mu_{4}(K)$.

As for line 11, we first consider the case $n=4$. As the automorphism group of the Dynkin diagram of $\mathrm{SL}_{2} \times \mathrm{SL}_{2} \times \mathrm{SL}_{2}$ is the symmetric group $\mathcal{S}_{3}$, we have the following result (compare [Hel12, §11]):

Corollary 9.10. Every linear transformation of $K^{2} \otimes K^{2} \otimes K^{2}$ that preserves the hyperdeterminant is of the form

$$
v_{1} \otimes v_{2} \otimes v_{3} \mapsto g_{1} v_{1} \otimes g_{2} v_{2} \otimes g_{3} v_{3} \quad \text { for } g_{1}, g_{2}, g_{3} \in \mathrm{GL}_{2}(K)
$$

such that $\operatorname{det}\left(g_{1} g_{2} g_{3}\right)= \pm 1$, or is the composition of such a map with a permutation

$$
v_{1} \otimes v_{2} \otimes v_{3} \mapsto v_{\sigma(1)} \otimes v_{\sigma(2)} \otimes v_{\sigma(3)} \quad \text { for } \sigma \in \mathcal{S}_{3} .
$$

Proof. In the notation of (2.5), one takes $\widetilde{L}$ to be a product of 3 copies of $\mathrm{GL}_{2}$ with the obvious $\widetilde{\rho}$; the kernel of $\widetilde{\rho}$ is isomorphic to $\mathbb{G}_{m} \times \mathbb{G}_{m}$. In view of Proposition 8.1 and $\$ 7$, it suffices to check that the permutations preserve the hyperdeterminant, which is clear from the explicit formula for the hyperdeterminant from, e.g., MW02.

For the representations on line 11 with $n \geq 5$, we define $\mathrm{GO}_{n}$ to be the algebraic group with $R$-points the matrices $g \in \mathrm{GL}_{n}(R)$ such that $g^{t} S g=\mu(g) S$ for some $\mu(g) \in R^{\times}$(for every $K$-algebra $R$ ); it is a reductive envelope of $\mathrm{O}_{n}$.

Corollary 9.11. For $n \geq 5$, every linear transformation of $M_{2 n}(K)$ that preserves the degree 4 function from Example 6.5 is of the form

$$
X \mapsto g_{1} X g_{2}^{t} \quad \text { for } g_{1} \in \mathrm{GL}_{2}(K), g_{2} \in \mathrm{GO}_{n}(K) \text { with } \operatorname{det}\left(g_{1}\right) \mu\left(g_{2}\right)= \pm 1 \text {. }
$$

Sketch of the proof. Note that $\operatorname{Aut}(\Delta, \lambda)$ is naturally identified with the component group of $\mathrm{GO}_{n}$.

As a concrete illustration of the remarks in $\$ 5$, we note that Corollary 9.11 and Example 6.5 go through with no change if we replace the split groups $\mathrm{SO}_{n}$ and $\mathrm{O}_{n}$ with the special orthogonal and orthogonal groups of any nondegenerate symmetric bilinear form, i.e., where the matrix $S$ in Example 6.5 is any symmetric invertible matrix. In this way, the corollary gives the stabilizer of $f$ also in the case where $K=\mathbb{R}$ and $\mathrm{SO}_{n}$ is replaced by a real group $\mathrm{SO}(2, n-2)$ or $\mathrm{SO}(6, n-6)$. This situation appears in the study of electromagnetic black hole charges in $\mathcal{N}=2$ or 4 supergravity; see e.g. $\mathrm{BDF}^{+} 12$. 


\section{Some representations omitted from table $\mathrm{A}$}

We have not yet discussed all pairs $(G, V)$ where $G$ is absolutely almost simple, $V$ is an irreducible representation of $G$, and $K[V]^{G}$ is generated by a homogeneous polynomial $f$. For $K=\mathbb{C}$, all such pairs are listed in the the table on pages 260-262 of PV94], and we now discuss each of the cases that we have thus far omitted.

Consider one of the groups $\mathrm{HSpin}_{n}$ for $n=7,9$ with their natural representations or $G_{2}$ with its 7 -dimensional representation. In these cases, $f$ has degree 2 , i.e., is a quadratic form, so its linear preserver is the orthogonal group $\mathrm{O}(f)$. Note that our Theorem 3.2 does not apply to these groups because they correspond to exclusions (1b) and (1c) in Definition 3.3 .

The natural 32-dimensional representation of the group HSpin $_{11}$ factors through the natural representation of $\mathrm{HSpin}_{12}$. The ring $K[V]^{\mathrm{HSpin}_{12}}$ is also 1-dimensional (as can be seen by the reasoning in $\sqrt[6]{6}$, where $H$ has type $E_{7}$ ) with generator $f$ of degree 4 , so clearly the $f$ stabilized by $\mathrm{HSpin}_{11}$ is the same as for $\mathrm{HSpin}_{12}$, and so the linear preserver of this $f$ is $\mathrm{HSpin}_{12} \cdot \mu_{4}$ as in Corollary 9.8, (For generalizations of this sort of example, see [Sol05].)

The only remaining pairs $(G, V)$ are $\left(\mathrm{SL}_{7}, \wedge^{3}\left(K^{7}\right)\right),\left(\mathrm{SL}_{8}, \wedge^{3}\left(K^{8}\right)\right)$, and $\mathrm{HSpin}_{14}$ with its natural 120-dimensional representation. The first representation is noteworthy, because the stabilizer in $\mathrm{SL}_{7}$ of any element $v$ such that $f(v) \neq 0$ is a group of type $G_{2}$; see for example Eng00, p. 65], Asc87b, or [CH88]. The orbits in the last representation have been studied over $\mathbb{C}$ in Pop80, and the fact that $\operatorname{dim} V / G=1$ has been applied to the theory of quadratic forms in Ros99a] and Ros99b; see also Gar09]. All three of these representations are irreducible and are not stable under an outer automorphism of $G$, so applying Theorem 3.2 . we find without doing any work that $\operatorname{Stab}_{\mathrm{GL}(V)}(\mathscr{O})$ is $\mathbb{G}_{m} \cdot G$. As to the preserver $\operatorname{Stab}_{\mathrm{GL}(V)}(f)$ in these cases, we omit serious investigation. However, for $K=\mathbb{C}$, one can observe that the identity component $G^{\prime}$ of $\operatorname{Stab}_{\mathrm{GL}(V)}(f)$ is reductive (because $V$ is an irreducible representation) and hence is semisimple (because the center must consist of scalar matrices). It follows from the classification of semisimple groups $G^{\prime}$ such that $\mathbb{C}[V]^{G^{\prime}}$ is generated by a single polynomial that $G^{\prime}=G$. In particular, $G$ is normal in $\operatorname{Stab}_{\mathrm{GL}(V)}(f)$. As $\operatorname{Aut}(\Delta, \lambda)=1$, it follows that $\operatorname{Stab}_{\mathrm{GL}(V)}(f)$ is contained in $G$. $\mathbb{G}_{m}$, i.e., $\operatorname{Stab}_{\mathrm{GL}(V)}(f)$ is $G . \mu_{d}$, where $d$ is the degree of $f$ (equal to 7,16 , or 8 respectively).

\section{An Alternative Formulation of the Linear PRESERVER PROBlem}

Inspecting the LPP solutions by Frobenius (1897) and Dieudonné (1949) where $V$ is the $n$-by- $n$ matrices and $f$ is the determinant, one sees that Frobenius determines the preserver of det, whereas Dieudonné determines the linear transformations on $V$ that preserve the set of singular matrices. So far, we have been solving Frobenius' version of the problem, but in fact we have also solved Dieudonné's version:

Proposition 11.1. For each of the representations in Table $\mathrm{A}$, the collection of linear transformations preserving the projective variety $f=0$ is $N_{\mathrm{GL}(V)}(G)$.

See e.g. Sch08 for general results on the relationship between the two versions.

Proof. Put $S$ for the sub-group-scheme of GL $(V)$ preserving the projective variety $f=0$. Given any $s \in S\left(K_{\text {alg }}\right),{ }^{s} f$ is in the ideal generated by $f$ and has the 
same degree as $f$; hence ${ }^{s} f=c f$ for some $c \in K^{\times}$and $c^{-1 / \operatorname{deg} f} s$ preserves $f$. Propositions 8.1 and 9.1 give that $s$ belongs to $N_{\mathrm{GL}(V)}(G)$.

Conversely, for $n \in \mathrm{GL}(V)$ normalizing $G$, Corollary 2.4 and Lemma 7.3 show that ${ }^{n} f$ is a scalar multiple of $f$.

Note that the proposition indeed solves Dieudonné's version of the linear preserver problem for the representations in Table A, because we calculated the group $N_{\mathrm{GL}(V)}(G)$ in 92

\section{ACKNOWLEDGEMENTS}

The authors thank Bob Guralnick, George McNinch, and Holger Petersson for inspiring conversations. The research for this article was partially supported by NSA grant no. H98230-11-1-0178.

\section{REFERENCES}

[ABS90] H. Azad, M. Barry, and G. Seitz, On the structure of parabolic subgroups, Comm. Algebra 18 (1990), no. 2, 551-562, DOI 10.1080/00927879008823931. MR.1047327 (91d:20048)

[Asc87a] Michael Aschbacher, The 27-dimensional module for E $E_{6}$. I, Invent. Math. 89 (1987), no. 1, 159-195, DOI 10.1007/BF01404676. MR892190 (88h:20045)

[Asc87b] Michael Aschbacher, Chevalley groups of type $G_{2}$ as the group of a trilinear form, J. Algebra 109 (1987), no. 1, 193-259, DOI 10.1016/0021-8693(87)90173-6. MR898346 (88g:20089)

$\left[\mathrm{BDF}^{+} 12\right]$ L. Borsten, M. J. Duff, S. Ferrara, A. Marrani, and W. Rubens, Small orbits, Phys. Rev. D 85 (2012), no. 8, 086002.

[Bea70] LeRoy B. Beasley, Linear transformations on matrices: The invariance of the third elementary symmetric function, Canad. J. Math. 22 (1970), 746-752. MR0268201 (42 \#3100)

[Bor91] Armand Borel, Linear algebraic groups, 2nd ed., Graduate Texts in Mathematics, vol. 126, Springer-Verlag, New York, 1991. MR.1102012 (92d:20001)

[Bou02] Nicolas Bourbaki, Lie groups and Lie algebras. Chapters 4-6, Elements of Mathematics (Berlin), Springer-Verlag, Berlin, 2002. Translated from the 1968 French original by Andrew Pressley. MR 1890629 (2003a:17001)

[Bou05] Nicolas Bourbaki, Lie groups and Lie algebras: Chapters 7-9, Springer-Verlag, Berlin, 2005. MR2109105

[Bro69] Robert B. Brown, Groups of type E $E_{7}$, J. Reine Angew. Math. 236 (1969), 79-102. MR 0248185 (40 \#1439)

[Cay45] A. Cayley, On the theory of linear transformations, Cambridge Mathematical Journal IV (1845), 193-209, (= Coll. Math. Papers, vol. 1, pp. 80-94).

[CG06] Skip Garibaldi and Michael Carr, Geometries, the principle of duality, and algebraic groups, Expo. Math. 24 (2006), no. 3, 195-234, DOI 10.1016/j.exmath.2005.11.001. MR2250947 (2007f:20080)

[CH88] Arjeh M. Cohen and Aloysius G. Helminck, Trilinear alternating forms on a vector space of dimension 7, Comm. Algebra 16 (1988), no. 1, 1-25, DOI 10.1080/00927878808823558. MR.921939 (89h:20060)

[Che97] Claude C. Chevalley, The algebraic theory of spinors, Columbia University Press, New York, 1954. MR0060497(15,678d)

[CL92] G. H. Chan and M. H. Lim, Linear transformations on symmetric matrices. II, Linear and Multilinear Algebra 32 (1992), no. 3-4, 319-325, DOI 10.1080/03081089208818172. MR.1238013(94i:15025)

[CLT87] Gin Hor Chan, Ming Huat Lim, and Kok-Keong Tan, Linear preservers on matrices, Linear Algebra Appl. 93 (1987), 67-80, DOI 10.1016/S0024-3795(87)90312-0. MR898543 (88h:15004)

[Dem77] M. Demazure, Automorphismes et déformations des variétés de Borel, Invent. Math. 39 (1977), no. 2, 179-186. MR.0435092 (55 \#8054) 
[DG70] M. Demazure and A. Grothendieck, SGA3: Schémas en groupes, Lecture Notes in Mathematics, vol. 151-153, Springer, 1970.

[Die49] Jean Dieudonné, Sur une généralisation du groupe orthogonal à quatre variables (French), Arch. Math. 1 (1949), 282-287. MR0029360 (10,5861)

[Eat69] Morris L. Eaton, On linear transformations which preserve the determinant, Illinois J. Math. 13 (1969), 722-727. MR0251050 (40 \#4281)

[Eng00] F. Engel, Ein neues, dem linearen Komplexe analoges Gebilde, Berichte über die Verhandlungen der Königlich Sächsischen Gesellschaft der Wissenschaften zu Leipzig. Mathematisch-Physische Klasse 52 (1900), 63-76, 220-239.

[ES72] Paul Eakin and James Silver, Rings which are almost polynomial rings, Trans. Amer. Math. Soc. 174 (1972), 425-449. MR0309924(46 \#9028)

[Fer72] J. C. Ferrar, Strictly regular elements in Freudenthal triple systems, Trans. Amer. Math. Soc. 174 (1972), 313-331 (1973). MR0374223 (51 \#10423)

[FH91] William Fulton and Joe Harris, Representation theory, Graduate Texts in Mathematics, vol. 129, Springer-Verlag, New York, 1991. A first course; Readings in Mathematics. MR.1153249 (93a:20069)

[Flo11] Mathieu Florence, On higher trace forms of separable algebras, Arch. Math. (Basel) 97 (2011), no. 3, 247-249, DOI 10.1007/s00013-011-0282-x. MR.2836304 (2012g:16034)

[Fro97] G. Frobenius, Über die Darstellung der endlichen Gruppen durch lineare Substitutionen, Sitzungsberichte Deutsch. Akad. Wiss. Berlin (1897), 994-1015.

[Gar04] Skip Garibaldi, The characteristic polynomial and determinant are not ad hoc constructions, Amer. Math. Monthly 111 (2004), no. 9, 761-778, DOI 10.2307/4145188. MR2104048 (2006d:15012)

[Gar09] Skip Garibaldi, Cohomological invariants: exceptional groups and spin groups, Mem. Amer. Math. Soc. 200 (2009), no. 937, xii+81. With an appendix by Detlev W. Hoffmann. MR 2528487 (2010g:20079)

[GGS02] Wee Teck Gan, Benedict Gross, and Gordan Savin, Fourier coefficients of modular forms on $G_{2}$, Duke Math. J. 115 (2002), no. 1, 105-169, DOI 10.1215/S0012-709402-11514-2. MR.1932327 (2004a:11036)

[Gre80] James A. Green, Polynomial representations of $\mathrm{GL}_{n}$, Lecture Notes in Mathematics, vol. 830, Springer-Verlag, Berlin, 1980. MR606556 (83j:20003)

[Gur64] G. B. Gurevich, Foundations of the theory of algebraic invariants, Translated by J. R. M. Radok and A. J. M. Spencer, P. Noordhoff Ltd., Groningen, 1964. MR0183733 $(32 \# 1211)$

[Gur97] Robert M. Guralnick, Invertible preservers and algebraic groups. II. Preservers of similarity invariants and overgroups of $\operatorname{PSL}_{n}(F)$, Linear and Multilinear Algebra 43 (1997), no. 1-3, 221-255, DOI 10.1080/03081089708818527. MR1613065 (99m:20108)

[Hel12] Fred W. Helenius, Freudenthal triple systems by root system methods, J. Algebra $\mathbf{3 5 7}$ (2012), 116-137, DOI 10.1016/j.jalgebra.2012.01.025. MR.2905245

[HM00] J. William Hoffman and Jorge Morales, Arithmetic of binary cubic forms, Enseign. Math. (2) 46 (2000), no. 1-2, 61-94. MR1769537 (2001h:11048)

[Hua48] Loo-Keng Hua, A theorem on matrices and its application to Grassmann space, Sci. Rep. Nat. Tsing Hua Univ. Ser. A. 5 (1948), 150-181. MR0030928 (11,75a)

[Hum80] J. E. Humphreys, Introduction to Lie algebras and representation theory, Graduate Texts in Mathematics, vol. 9, Springer-Verlag, 1980, Third printing, revised.

[Hum81] _ Linear algebraic groups, second ed., Graduate Texts in Mathematics, vol. 21, Springer, 1981.

[Igu70] Jun-ichi Igusa, A classification of spinors up to dimension twelve, Amer. J. Math. 92 (1970), 997-1028. MR.0277558 (43 \#3291)

[Jac61] N. Jacobson, Some groups of transformations defined by Jordan algebras. III, J. Reine Angew. Math. 207 (1961), 61-85. MR0159850(28 \#3066)

[Jac68] N. Jacobson, Structure and representations of Jordan algebras, Coll. Pub., vol. 39, Amer. Math. Soc., Providence, RI, 1968. MR0251099

[Jan03] Jens Carsten Jantzen, Representations of algebraic groups, 2nd ed., Mathematical Surveys and Monographs, vol. 107, American Mathematical Society, Providence, RI, 2003. MR2015057 (2004h:20061)

[Kac80] V. G. Kac, Some remarks on nilpotent orbits, J. Algebra 64 (1980), no. 1, 190-213, DOI 10.1016/0021-8693(80)90141-6. MR.575790 (81i:17005) 
[KM78] T. Kambayashi and M. Miyanishi, On flat fibrations by the affine line, Illinois J. Math. 22 (1978), no. 4, 662-671. MR503968 (80f:14028)

[KMRT98] Max-Albert Knus, Alexander Merkurjev, Markus Rost, and Jean-Pierre Tignol, The book of involutions, American Mathematical Society Colloquium Publications, vol. 44, American Mathematical Society, Providence, RI, 1998. With a preface in French by J. Tits. MR.1632779(2000a:16031)

[Lim79] M. H. Lim, Linear transformations on symmetric matrices, Linear and Multilinear Algebra 7 (1979), no. 1, 47-57, DOI 10.1080/03081087908817259. MR523648(80f:15027)

[LP01] Chi-Kwong Li and Stephen Pierce, Linear preserver problems, Amer. Math. Monthly 108 (2001), no. 7, 591-605, DOI 10.2307/2695268. MR1862098 (2002g:15005)

[LT92] Chi-Kwong $\mathrm{Li}$ and Nam-Kiu Tsing, Linear preserver problems: a brief introduction and some special techniques, Linear Algebra Appl. 162/164 (1992), 217-235, DOI 10.1016/0024-3795(92)90377-M. Directions in matrix theory (Auburn, AL, 1990). MR:1148401 (93b:15003)

[Lur01] Jacob Lurie, On simply laced Lie algebras and their minuscule representations, Comment. Math. Helv. 76 (2001), no. 3, 515-575, DOI 10.1007/PL00013217. MR.1854697 (2002g:17015)

[Mar62] Marvin Marcus, Linear operations on matrices, Amer. Math. Monthly 69 (1962), 837-847. MR0147491 (26 \#5007)

[Mey68] Kurt Meyberg, Eine Theorie der Freudenthalschen Tripelsysteme. I, II (German), Nederl. Akad. Wetensch. Proc. Ser. A 71=Indag. Math. 30 (1968), 162-174, 175-190. MR 0225838 (37 \#1429)

[Min77] Henryk Minc, Linear transformations on matrices: rank 1 preservers and determinant preservers, Linear and Multilinear Algebra 4 (1976/77), no. 4, 265-272. MR 0435098 (55 \#8060)

[MM59a] Marvin Marcus and B. N. Moyls, Linear transformations on algebras of matrices, Canad. J. Math. 11 (1959), 61-66. MR0099996 (20 \#6432)

[MM59b] Marvin Marcus and B. N. Moyls, Transformations on tensor product spaces, Pacific J. Math. 9 (1959), 1215-1221. MR0108503 (21 \#7219)

[MP59] Marvin Marcus and Roger Purves, Linear transformations on algebras of matrices: the invariance of the elementary symmetric functions, Canad. J. Math. 11 (1959), 383-396. MR0105425 (21 \#4167)

[MW60] Marvin Marcus and Roy Westwick, Linear maps on skew symmetric matrices: the invariance of elementary symmetric functions, Pacific J. Math. 10 (1960), 917-924. MR0114823 (22 \#5641)

[MW02] Akimasa Miyake and Miki Wadati, Multipartite entanglement and hyperdeterminants, Quantum Inf. Comput. 2 (2002), no. suppl., 540-555. ERATO Workshop on Quantum Information Science (Tokyo, 2002). MR1975158 (2004e:81028)

[Nem63] William C. Nemitz, Transformations preserving the Grassmannian, Trans. Amer. Math. Soc. 109 (1963), 400-410. MR0154882 (27 \#4826)

[PD95] Vladimir P. Platonov and Dragomir Z. Đoković, Linear preserver problems and algebraic groups, Math. Ann. 303 (1995), no. 1, 165-184, DOI 10.1007/BF01460985. MR $1348361(96 \mathrm{~m}: 20072)$

$\left[\mathrm{PLL}^{+}\right.$92] S. Pierce, M. H. Lim, R. Loewy, C.K. Li, N.K. Tsing, B. McDonald, and L. Beasley, A survey of linear preserver problems, Gordon and Breach Science Publishers, Yverdon, 1992, Linear and Multilinear Algebra 33, no. 1-2. MR 1346777 (96c:15043)

[Pop80] V. L. Popov, Classification of spinors of dimension 14, Trans. Moscow Math. Soc. (1980), no. 1, 181-232.

[PV94] V. L. Popov and E. B. Vinberg, Invariant theory, Encyclopedia of Mathematical Sciences, vol. 55, pp. 123-284, Springer-Verlag, 1994.

[Rev79] Philippe Revoy, Trivecteurs de rang 6 (French), Bull. Soc. Math. France Mém. 59 (1979), 141-155. Colloque sur les Formes Quadratiques, 2 (Montpellier, 1977). MR532012 (80k:15041)

[Röh93] Gerhard E. Röhrle, On extraspecial parabolic subgroups, Linear algebraic groups and their representations (Los Angeles, CA, 1992), Contemp. Math., vol. 153, Amer. Math. Soc., Providence, RI, 1993, pp. 143-155, DOI 10.1090/conm/153/01310. MR 1247502 (94k:20082) 
[Ros99a] M. Rost, On 14-dimensional quadratic forms, their spinors, and the difference of two octonion algebras, unpublished note, March 1999.

[Ros99b] On the Galois cohomology of Spin(14), unpublished note, March 1999.

[RRS92] Roger Richardson, Gerhard Röhrle, and Robert Steinberg, Parabolic subgroups with abelian unipotent radical, Invent. Math. 110 (1992), no. 3, 649-671, DOI 10.1007/BF01231348. MR.1189494 (93j:20092)

[Rub92] Hubert Rubenthaler, Algèbres de Lie et espaces préhomogènes (French), Travaux en Cours [Works in Progress], vol. 44, Hermann Éditeurs des Sciences et des Arts, Paris, 1992. With a foreword by Jean-Michel Lemaire. MR 2412335 (2009e:20101)

[Sch00] A. Schinzel, Polynomials with special regard to reducibility, Encyclopedia of Mathematics and its Applications, vol. 77, Cambridge University Press, Cambridge, 2000. With an appendix by Umberto Zannier. MR1770638 (2001h:11135)

[Sch08] Gerald W. Schwarz, Linear maps preserving fibers, J. Lie Theory 18 (2008), no. 2, 433-443. MR2431126(2009d:20111)

[Sei87] Gary M. Seitz, The maximal subgroups of classical algebraic groups, Mem. Amer. Math. Soc. 67 (1987), no. 365, iv+286. MR888704 (88g:20092)

[Ses77] C. S. Seshadri, Geometric reductivity over arbitrary base, Advances in Math. 26 (1977), no. 3, 225-274. MR0466154 (57 \#6035)

[Sha83] Ronald Shaw, Linear algebra and group representations. Vol. II, Academic Press Inc. [Harcourt Brace Jovanovich Publishers], London, 1983. Multilinear algebra and group representations. MR701854 (84m:15003)

[Sin83] Richard Sinkhorn, Linear adjugate preservers on the complex matrices, Linear and Multilinear Algebra 12 (1982/83), no. 3, 215-222, DOI 10.1080/03081088208817485. MR678827 (84a:15002)

[SK77] M. Sato and T. Kimura, A classification of irreducible prehomogeneous vector spaces and their relative invariants, Nagoya Math. J. 65 (1977), 1-155. MR.0430336 (55 \#3341)

[Sol05] S. Solomon, Irreducible linear group-subgroup pairs with the same invariants, J. Lie Theory 15 (2005), no. 1, 105-123. MR2115231(2005j:13008)

[Spr06] T. A. Springer, Some groups of type E $E_{7}$, Nagoya Math. J. 182 (2006), 259-284. MR2235344(2007b:20096)

[SV00] Tonny A. Springer and Ferdinand D. Veldkamp, Octonions, Jordan algebras and exceptional groups, Springer Monographs in Mathematics, Springer-Verlag, Berlin, 2000. MR.1763974 (2001f:17006)

[Wat79] William C. Waterhouse, Introduction to affine group schemes, Graduate Texts in Mathematics, vol. 66, Springer-Verlag, New York, 1979. MR.547117 (82e:14003)

[Wat82] William C. Waterhouse, Linear maps preserving reduced norms, Linear Algebra Appl. 43 (1982), 197-200, DOI 10.1016/0024-3795(82)90253-1. MR656445 (83i:16025)

[Wat83] William C. Waterhouse, Invertibility of linear maps preserving matrix invariants, Linear and Multilinear Algebra 13 (1983), no. 2, 105-113, DOI 10.1080/03081088308817510. MR697321 (84i:15020)

[Wat87] William C. Waterhouse, Automorphisms of $\operatorname{det}\left(X_{i j}\right)$ : the group scheme approach, Adv. in Math. 65 (1987), no. 2, 171-203, DOI 10.1016/0001-8708(87)90021-1. MR 900267 (88k:14025)

[Wat89] William C. Waterhouse, Linear transformations preserving symmetric rank one matrices, J. Algebra 125 (1989), no. 2, 502-518, DOI 10.1016/0021-8693(89)90179-8. MR.1018960 (91d:16059)

[Wat95] William C. Waterhouse, Automorphism group schemes of basic matrix invariants, Trans. Amer. Math. Soc. 347 (1995), no. 10, 3859-3872, DOI 10.2307/2155207. MR:1303128 (96c:14036)

[Web95] H. Weber, Lehrbuch der algebra, Friedrich Vieweg und Sohn, 1895.

[Wes64] R. Westwick, Linear transformations on Grassman spaces, Pacific J. Math. 14 (1964), 1123-1127. MR0167493 (29 \#4766)

[Wes67] Roy Westwick, Transformations on tensor spaces, Pacific J. Math. 23 (1967), 613-620. MR0225805 (37 \#1397)

[Wes69] Roy Westwick, Linear transformations on Grassmann spaces, Canad. J. Math. 21 (1969), 414-417. MR0241459 (39 \#2799) 
[Zha05] Xian Zhang, Linear/additive preservers of rank 2 on spaces of alternate matrices over fields, Linear Algebra Appl. 396 (2005), 91-102, DOI 10.1016/j.laa.2004.08.028. MR2112201 (2006a:15011)

Department of Mathematics and Computer Science, MSC W401, Emory University, 400 Dowman Drive, Atlanta, Georgia 30322

Department of Mathematics and Computer Science, MSC W401, Emory University, 400 Dowman Drive, Atlanta, Georgia 30322

Department of Mathematics and Computer Science, MSC W401, Emory University, 400 Dowman Drive, Atlanta, Georgia 30322 